Journal of Clinical Investigation

Vol. 41, No. 4, 1962

\title{
PATTERNS OF HUMAN MYOCARDIAL OXYGEN EXTRACTION DURING REST AND EXERCISE *
}

\author{
By JOSEPH V. MESSER, RICHARD J. WAGMAN, HERBERT J. LEVINE, \\ WILLIAM A. NEILL, NORMAN KRASNOW AND RICHARD GORLIN †
}

(From the Peter Bent Brigham Hospital, Boston, Mass.)

(Submitted for publication July 27, 1961 ; accepted November 30, 1961)

Myocardial oxygen requirements are met by coronary blood flow and oxygen extraction from the arterial blood. The myocardial oxygen extraction coefficient (1) expresses the fraction of available oxygen in arterial blood removed by the myocardium : coronary $\mathrm{A}-\mathrm{Vo}_{2} / \mathrm{Ao}_{2} \times 100=$ myocardial extraction percentage. Myocardial oxygen extraction during rest is approximately 70 to 75 per cent, exceeding that of all other organs $(1,2)$. This degree of extraction is equalled only by exercising skeletal muscle, in which increased oxygen requirements are met both by additional blood flow and by increased oxygen extraction. Earlier workers have noted a relative constancy of myocardial oxygen extraction under the widely varying conditions of rest, anemia, hypoxia, and increased left ventricular work, suggesting that coronary blood flow is altered commensurate with myocardial oxygen requirements $(1,3,4)$. Case, Berglund and Sarnoff demonstrated that when coronary blood flow reserve was limited by experimental coronary constriction, myocardial extraction increased due to the combined stress of anemia and increased left ventricular work (3).

The effect of exercise on coronary hemodynamics has received little attention. Lombardo and co-workers reported observations in $13 \mathrm{hu}$ man subjects with various forms of heart disease (5). As part of another study, Regan and associates reported on the effects of exercise on the coronary circulation in nine normal subjects (6). This report will present myocardial oxygen extraction data from 108 patients at rest, and from 79 subjects during mild exercise. The physiological significance of myocardial oxygen extrac-

* This work was supported by grants from the U. S. Public Health Service (H-2637), Massachusetts Heart Association (no. 390), and Kriendler Memorial Foundation, New York, N. Y.

† Investigator, Howard Hughes Medical Institute. tion data and their correlation with various clinical states will form the basis of this communication.

\section{MATERIALS AND METHODS}

Eighty observations were made at rest and during exercise in 79 patients referred to the Peter Bent Brigham Hospital for diagnostic cardiac catheterization. Thirty additional resting observations were made in 29 patients. Clinical diagnoses in these patients were based on history, physical and fluoroscopic examination, resting electrocardiogram, the standard two-step test of Master (7), and routine cardiac catheterization findings. The exercise electrocardiograms were interpreted according to the criteria of Mattingly, Fancher, Bauer and Robb (8).

Four clinical states were selected for correlation with myocardial oxygen extraction patterns. The patients were grouped clinically according to the following criteria.

Control. Patients having no symptoms or electrocardiographic, radiologic or physical findings suggesting coronary artery disease or congestive heart failure served as controls. Pulmonary capillary pressures were normal in all subjects except those with mild mitral stenosis. The range of diagnoses included functional systolic murmurs, patent ductus arteriosus, ventricular and atrial septal defects, mild aortic insufficiency, noncritical mitral stenosis (valve area greater than $1.5 \mathrm{~cm}^{2}$ ), tetralogy of Fallot, and mild coarctation of the aorta. Two had atypical chest pain without evidence to suggest coronary insufficiency.

Coronary insufficiency. Patients judged to have coronary insufficiency on the basis of classical angina pectoris, documented myocardial infarction, and positive Master's test were placed in this group. In several cases the coronary vessels were assessed at operation, by coronary arteriography (9), or at necropsy. Several patients had coexisting congestive heart failure.

Congestive heart failure. This group consisted of patients with typical signs and symptoms of left ventricular failure, documented by elevated left ventricular filling pressures.

Mitral stenosis. These were patients having mitral stenosis of critical degree with valve areas of less than $1.5 \mathrm{~cm}^{2}$.

The diagnosis of each subject appears with other pertinent clinical data in Tables II, III, and IV. In addition, the presence or absence of coronary insufficiency 


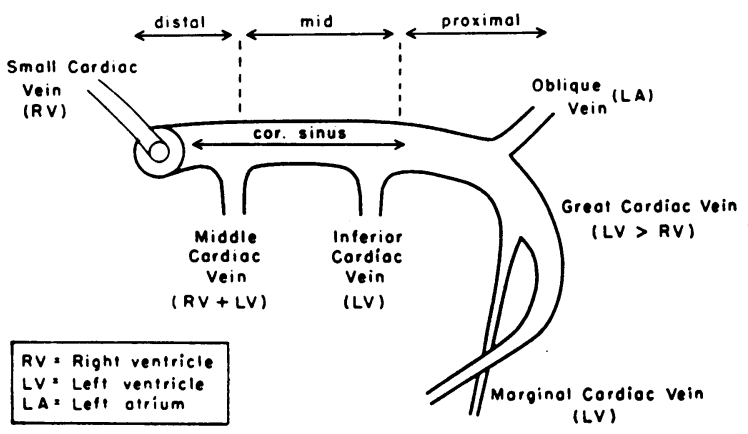

Fig. 1. Dominant Pattern FOR CORONARy venous DRAINAGE $(17,18)$. The principal sources of blood draining to each cardiac vein are shown in parentheses. $\mathrm{RV}=$ right ventricle; $L V=$ left ventricle; $L A=$ left atrium. The proximal coronary sinus drains principally left ventricular blood (19). According to James, the middle cardiac vein has also been termed "posterior interventricular vein" and the inferior cardiac vein termed the "left posterior interventricular vein" (19).

and congestive heart failure is indicated for each patient.

The study was performed during the course of routine diagnostic right heart catheterization. Left heart catheterization was done whenever necessary to determine left ventricular dynamics. Patients were fasted and, in most instances, unsedated. A catheter was placed in the brachial artery or aorta by the Seldinger technic (10). A second catheter of the Goodale-Lubin type was introduced into the proximal coronary sinus ${ }^{1}$ (Figure 1). Arterial and coronary venous blood samples and pressures were obtained from these sites in the 80 rest-exer-

1 Anatomically, the proximal coronary sinus arises from the confluence of the great cardiac and oblique veins, approximately 2 to 3 inches from the right atrial ostium of the sinus. cise observations, according to the protocol presented in Figure 2. During the early phase of this study, single resting measurements were made 10 minutes after exercise in the belief that these values represented a more basal state than before exercise. Thereafter, resting observations before and after exercise were made, and revealed no statistically significant difference between the resting states $(p>0.5)$. These findings are in accord with those of Dexter (11) and Donald (12) and their co-workers, who demonstrated the reproducibility of resting measurements before and after exercise. After assessment of the coronary circulation, the venous catheter was advanced to the pulmonary artery and cardiac outputs and pressures were measured during similar exercise and rest postexercise periods. Peripheral hemodynamics were reproducible during the two exercise periods for coronary circulatory and cardiac output determinations. In the majority of subjects the direct Fick method was used, while in some, the $\mathrm{I}^{131}$-albumin indicator dilution technic was employed. In some subjects cardiac output was measured before, and in others, before and after coronary venous catheterization. No systematic difference was noted in relation to the time of cardiac output measurement; the time elapsed between exercise periods was approximately 20 minutes. Pulmonary capillary pressures, when measured, were obtained during the cardiac output periods. Lead II electrocardiograms were obtained at rest and immediately after exercise. Exercise for coronary and systemic measurements was identical and consisted of approximately 8 minutes of supine leg-raising sufficient to increase total body oxygen consumption two- to three-fold over the resting value. This degree of exercise increased total body oxygen consumption approximately one-half as much as the standard Master's test (13).

All pressures were measured with a Statham P-23D manometer. Pressures and electrocardiograms were recorded directly on a Sanborn four-channel recorder. Systemic and pulmonary arterial and coronary venous oxygen contents and capacities were determined in du-

\begin{tabular}{l|c|cc|c|c|} 
& $\begin{array}{c}\text { REST } \\
\text { PRE-EXERCISE }\end{array}$ & $\begin{array}{ccc}\text { EXERCISE TIME } \\
3^{\prime}\end{array}$ & $7^{\prime}$ & RECOVERY TIME & $\begin{array}{c}\text { REST } \\
\text { POST-EXECISE } \\
\text { 10-20' }\end{array}$ \\
\hline ELECTROCARDIOGRAM (Ld II) & $\mathrm{x}$ & & & $\mathrm{x} \times \mathrm{x}$ & $\mathrm{x}$ \\
BA + CS PRESSURE & $\mathrm{x}$ & $\mathrm{x}$ & $\mathrm{x}$ & & $\mathrm{x}$ \\
BA + CS OXYGEN SAMPLE & $\mathrm{x}$ & $\mathrm{x}$ & $\mathrm{x}$ & & $\mathrm{x}$ \\
\hline
\end{tabular}

$$
\begin{aligned}
& X=\text { Observation or Blood Sample } \\
& B A=\text { Brachial Artery } \\
& C S=\text { Coronary Sinus }
\end{aligned}
$$

Fig. 2. Protocol of Rest-exercise study. Observations were made according to the time schedule shown above. $\mathrm{BA}=$ brachial artery; $\mathrm{CS}=$ coronary sinus. 
plicate according to the method of van Slyke and Neill (14). Oxygen extraction percentages were calculated for the myocardium and total body.

In order to assess the homogeneity of cardiac venous blood, duplicate samples were obtained in 18 subjects from distal, middle, and proximal sites as shown in Figure 1. Oxygen saturation was determined in eight subjects using the Beckman model DU spectrophotometer, and oxygen content was calculated from resting oxygen capacity. The van Slyke technic was employed in the remaining ten subjects. To insure uniformity of sampling during rest and exercise, the catheter position within the coronary sinus was examined fluoroscopically during and after each study period. ${ }^{2}$ Statistical analyses were determined using the $t$ distribution of Snedecor (15).

\section{RESULTS}

\section{Homogeneity of coronary venous drainage}

The oxygen saturation and content of blood from three sites within the coronary sinus are

2 The catheter was inserted into the coronary sinus in the right anterior oblique position and the patient returned to the supine position. The catheter was advanced in a cephalad direction to approximately 1 to 1.5 inches from the left lateral border of the heart, a position judged to be in the proximal coronary sinus (Figure 1). The blood obtained was of low saturation, the pressure was at a venous level, and the catheter had to remain unchanged in position from the beginning to the end of the procedure. shown in Table I. Six control subjects and 12 coronary insufficiency subjects are included. With one exception (TL) no significant difference in oxygen saturation or content was found between the proximal and middle portions of the coronary sinus $(p=0.5)$. This was observed over a wide range of oxygen saturations ( 18 to 42 per cent), and irrespective of the presence or absence of coronary artery disease. In one patient (JP) blood from the middle cardiac vein had a significantly higher oxygen saturation than the more proximal samples. Blood obtained distal to the junction of the middle and great cardiac veins was sometimes more highly saturated, probably reflecting middle cardiac venous and right atrial contamination.

\section{Physiological observations}

Tables II, III and IV show 110 resting observations in 108 subjects. In all but four subjects (MR, LR, RH, MM) arterial oxygen saturation was 91 per cent or greater. A wide range of coronary venous oxygen contents and saturations and of myocardial oxygen extraction percentages was observed among the subjects.

TABLE I

Oxygen mapping

\begin{tabular}{|c|c|c|c|c|c|c|c|c|c|}
\hline & \multirow[b]{3}{*}{ Patient } & \multicolumn{8}{|c|}{ Great coronary vein } \\
\hline & & \multicolumn{2}{|c|}{ Proximal } & \multicolumn{2}{|c|}{ Mid } & \multicolumn{2}{|c|}{ Distal } & \multicolumn{2}{|c|}{ "Out" } \\
\hline & & Content & Sat.* & Content & Sat. & Content & Sat. & Content & Sat. \\
\hline \multirow{16}{*}{$\begin{array}{l}\text { Control } \\
\text { Coronary disease }\end{array}$} & \multirow{16}{*}{$\begin{array}{l}\text { JM } \\
\text { LT } \\
\text { DB } \\
\text { JL } \\
\text { MM } \\
\text { TL } \\
\text { SB } \\
\text { RC } \\
\text { YSt } \\
\text { MC } \\
\text { BS } \\
\text { ST } \\
\text { WM } \\
\text { EB } \\
\text { LN } \\
\text { JP } \\
\text { EE } \\
\text { JL }\end{array}$} & vol \% & $\%$ & vol \% & $\%$ & vol \% & $\%$ & vol \% & $\%$ \\
\hline & & \multirow{15}{*}{$\begin{array}{l}5.8 \\
9.1 \\
7.1 \\
3.8 \\
3.5 \\
8.5 \\
5.1 \\
6.0 \\
4.4 \\
3.1 \\
4.7 \\
4.9 \\
8.0 \\
4.0 \\
6.7 \\
7.2 \\
7.3 \\
5.8\end{array}$} & \multirow{15}{*}{$\begin{array}{l}22 \\
40.5 \\
38 \\
19 \\
22 \\
42 \\
30 \\
30 \\
24 \ddagger \\
19 \\
24 \\
26 \\
38 \\
26 \\
38 \\
37 \\
35 \\
33\end{array}$} & \multirow{15}{*}{$\begin{array}{l}5.6 \\
9.0 \\
6.9 \\
4.1 \\
3.4 \\
9.5 \\
4.4 \\
6.4 \\
4.1 \\
2.9 \\
5.1 \\
4.7 \\
7.9 \\
3.9 \\
7.1 \\
8.8 \\
7.3 \\
5.9\end{array}$} & \multirow{15}{*}{$\begin{array}{l}21 \\
40 \\
36 \\
20.5 \\
21 \\
47 \\
27 \\
32 \\
22 \\
18 \\
26.5 \\
25 \\
37 \\
25 \\
40 \\
45 \S \\
35 \\
33\end{array}$} & & & \multirow{4}{*}{6.1} & \multirow{4}{*}{30.5} \\
\hline & & & & & & & & & \\
\hline & & & & & & 9.3 & 50 & & \\
\hline & & & & & & 3.9 & 24 & & \\
\hline & & & & & & 8.7 & $43 \dagger$ & \multirow[t]{2}{*}{13.9} & \multirow[t]{2}{*}{69} \\
\hline & & & & & & 5.1 & 30 & & \\
\hline & & & & & & 6.4 & 32 & \multirow[t]{3}{*}{10.0} & \multirow[t]{3}{*}{51} \\
\hline & & & & & & 5.8 & 31 & & \\
\hline & & & & & & 3.0 & 19 & & \\
\hline & & & & & & & & \multirow[t]{2}{*}{8.0} & \multirow[t]{2}{*}{41} \\
\hline & & & & & & 4.7 & 25 & & \\
\hline & & & & & & & & \multirow[t]{2}{*}{9.1} & \multirow[t]{2}{*}{43} \\
\hline & & & & & & 4.1 & 26 & & \\
\hline & & & & & & $\begin{array}{l}7.2 \\
7.2\end{array}$ & $\begin{array}{l}41 \\
37\end{array}$ & \multirow{2}{*}{14.3} & \multirow[t]{2}{*}{73} \\
\hline & & & & & & 7.7 & 37 & & \\
\hline
\end{tabular}

* Saturation.

$+1 \mathrm{~cm}$ distal to mid sample.

f Anterior interventricular vein.

$\$$ Probably middle cardiac vein. 
TABLE

\begin{tabular}{|c|c|c|c|c|c|c|c|c|c|c|c|}
\hline \multirow[b]{3}{*}{ No. } & \multirow[b]{3}{*}{$\mathrm{Pt}$} & \multirow[b]{3}{*}{ Sex } & \multirow[b]{3}{*}{ Age } & \multirow[b]{3}{*}{ BSA } & \multirow[b]{3}{*}{ Diagnosis } & \multirow[b]{3}{*}{ CI } & \multirow[b]{3}{*}{$\mathrm{CHF}$} & \multicolumn{4}{|c|}{ Brachial artery $O=$ content } \\
\hline & & & & & & & & \multirow{2}{*}{$\begin{array}{l}\text { Rest } \\
\text { Pre- } \\
\text { exer- } \\
\text { cise- }\end{array}$} & \multicolumn{2}{|c|}{ Exercise } & \multirow{2}{*}{$\begin{array}{l}\text { Rest } \\
\text { Post- } \\
\text { exer- } \\
\text { cise }\end{array}$} \\
\hline & & & & & & & & & $3 \min$ & $7 \mathrm{~min}$ & \\
\hline & & & yrs & $m^{2}$ & & & & \multicolumn{2}{|c|}{ vol \% } & \multicolumn{2}{|c|}{ vol \% } \\
\hline 1 & BH & f & 32 & 1.4 & FSM & 0 & 0 & & 15.2 & $14 . t$ & 14.1 \\
\hline 2 & PA & f & 29 & 1.58 & FSM & 0 & $\mathbf{0}$ & 15.7 & 16.2 & 16.3 & 15.9 \\
\hline 3 & $\mathrm{CH}$ & $\mathrm{m}$ & 31 & 2.00 & $\mathrm{CA}$, mild & 0 & 0 & 19.5 & 20.3 & 20.6 & 19.4 \\
\hline 4 & RL & f & 53 & 1.65 & MS, mild (valve area $2 \mathrm{~cm}^{2}$ ) & 0 & 0 & & 17.7 & & 17.0 \\
\hline 5 & PR & $\mathrm{f}$ & 27 & 1.5 & MS, mild (valve area $>3 \mathrm{~cm}^{2}$ ) & 0 & 0 & & 18.0 & 17.3 & 17.4 \\
\hline 6 & RM & $\mathrm{f}$ & 42 & 1.68 & MS, mild (valve area $2.2 \mathrm{~cm}^{2}$ ) & 0 & $\mathbf{0}$ & & 15.2 & & 14.7 \\
\hline 7 & YA & $\mathrm{f}$ & 40 & 1.62 & MS, mild (valve area $2.8 \mathrm{~cm}^{2}$ ) & $\mathbf{0}$ & 0 & 15.3 & 15.4 & 15.0 & 14.6 \\
\hline 8 & $\mathrm{JC}$ & f & 28 & 1.28 & AI, mild & $\mathbf{0}$ & 0 & 14.3 & 14.5 & & \\
\hline 9 & LS & f & 29 & 1.58 & AI, mild & $\mathbf{0}$ & 0 & & 17.1 & & 16.7 \\
\hline 10 & WG & $\mathrm{m}$ & 39 & 2.12 & AI, mild & $\mathbf{0}$ & $\mathbf{0}$ & 18.1 & 19.4 & 19.4 & 18.3 \\
\hline 11 & RG & $\mathrm{m}$ & 25 & 1.68 & AI, moderate & 0 & $\mathbf{0}$ & 19.9 & 20.0 & 19.5 & 19.3 \\
\hline 12 & JD & $\mathrm{m}$ & 57 & 1.80 & AI, moderate & $\mathbf{0}$ & 0 & 19.7 & 19.9 & 19.9 & 19.7 \\
\hline 13 & MS & $\mathrm{m}$ & 49 & 1.75 & AI, moderate & 0 & 0 & 20.0 & & 21.1 & \\
\hline 14 & MM & f & 31 & 1.65 & PDA ; $\mathrm{p} / \mathrm{s}$ shunt ratio 1.2 & 0 & 0 & 14.0 & & 15.1 & \\
\hline 15 & MT & $\mathrm{f}$ & 45 & 1.56 & $\mathrm{PDA} ; \mathrm{p} / \mathrm{s}$ shunt ratio 1.7 & 0 & 0 & 16.8 & 16.7 & & 17.0 \\
\hline 16 & $\mathrm{CL}$ & $\mathrm{f}$ & 37 & $1: 27$ & PDA; $\mathrm{p} / \mathrm{s}$ shunt ratio 1.5 & 0 & 0 & 17.5 & 18.1 & 18.0 & 17.4 \\
\hline 17 & WC & $\mathrm{m}$ & 25 & 1.9 & $\mathrm{PDA} ; \mathrm{p} / \mathrm{s}$ shunt ratio 1.5 & 0 & 0 & 17.0 & 17.7 & & \\
\hline 18 & AA & $\mathrm{f}$ & 16 & 1.56 & $\begin{array}{l}\text { VSD; } p / s \text { shunt ratio } 2.3 \\
\text { NCA (op.) }\end{array}$ & 0 & 0 & & 17.7 & & 17.4 \\
\hline 19 & MR & f & 38 & 1.62 & $\mathrm{~T}$ of $\mathrm{F} ; \mathrm{NCA}$ (P.M.) & 0 & 0 & 11.5 & 9.9 & 9.8 & \\
\hline 20 & EL & $\mathrm{m}$ & 31 & 1.6 & $\mathrm{~T}$ of $\mathrm{F}$ & 0 & 0 & 19.9 & 17.9 & 16.1 & 19.4 \\
\hline 21 & FS & $\mathbf{m}$ & 40 & 1.80 & ÂS (valve area $0.9 \mathrm{~cm}^{2}$ ); NCA (op.) & 0 & 0 & & 17.8 & 18.9 & 18.5 \\
\hline 22 & SP & $\mathrm{m}$ & 45 & 1.97 & AS (valve area $\left.1.0 \mathrm{~cm}^{2}\right)$ & 0 & 0 & & 17.1 & & 17.2 \\
\hline 23 & ML & $\mathrm{m}$ & 19 & 1.75 & $\mathrm{SS}$, mild (gradient $22 \mathrm{~mm} \mathrm{Hg}$ ) & 0 & 0 & 19.1 & 18.8 & 19.0 & 19.0 \\
\hline 24 & $\mathrm{CC}$ & $\mathrm{f}$ & 64 & 1.50 & ASD; $p / s$ shunt ratio $2.5 ; \mathrm{SH}$, mild & 0 & 0 & 15.9 & 15.7 & 15.8 & 15.6 \\
\hline 25 & AY & $\mathrm{m}$ & 21 & 2.05 & $\begin{array}{l}\text { Grade } 2 \text { SM (LVH by voltage cri- } \\
\text { teria only) }\end{array}$ & 0 & 0 & & 20.7 & 21.0 & 20.3 \\
\hline 26 & $\mathrm{JL}$ & $\mathrm{m}$ & 43 & 2.05 & Fatigue & 0 & 0 & 18.8 & 19.5 & 19.9 & 19.5 \\
\hline 27 & ES & f & 46 & 1.7 & Probable $\mathrm{PDA} ; \mathrm{p} / \mathrm{s}$ shunt ratio 1.5 & $\mathbf{0}$ & 0 & 17.4 & & 17.8 & \\
\hline 28 & TM & $\mathrm{m}$ & 45 & 1.82 & Atypical chest pain ; negative EKG & 0 & $\mathbf{0}$ & & 19.7 & & 19.1 \\
\hline 29 & LT & $\mathrm{m}$ & 20 & 1.88 & $\begin{array}{l}\text { Grade } 3 \mathrm{SM} \text {; negative diagnostic } \\
\text { studies; biphasic } T \text { waves, leads }\end{array}$ & 0 & 0 & 21.5 & 21.8 & 21.9 & 21.9 \\
\hline 30 & Sbi & $\mathrm{f}$ & 34 & 1.59 & Atypical chest pain; negative EKG & 0 & 0 & 16.0 & 16.0 & 16.5 & 15.9 \\
\hline 31 & DH & $\mathrm{m}$ & 54 & 1.58 & AS; NCA (op.) & + & 0 & 20.3 & 20.1 & 19. & 20.1 \\
\hline 32 & $\mathrm{SB}$ & $\mathrm{f}$ & 36 & 1.54 & CAD; ACA (op.) & + & 0 & 16.5 & 16.9 & 16.9 & 16.6 \\
\hline 33 & LY & f & 37 & 1.65 & Probable CAD; LVH & + & 0 & 15.1 & 15.5 & 15.0 & 15.0 \\
\hline 34 & HR & $\mathrm{m}$ & 61 & 1.82 & $\mathrm{CAD} ; \mathrm{MI}$ & + & + & & 18.4 & & 18.2 \\
\hline 35 & LR & $\mathrm{m}$ & 53 & 1.70 & AS; NCA (op. \& P.M.) & + & + & 14.5 & 14.8 & 15.5 & 13.4 \\
\hline 36 & DS & $\mathbf{m}$ & 31 & 1.70 & AI; NCA (op.) & 0 & + & 17.0 & 17.5 & 17.9 & \\
\hline 37. & $\mathrm{JF}$ & $\mathrm{m}$ & 52 & 1.54 & SS; NCA (op.) & $\mathbf{0}$ & + & 15.3 & 15.5 & & \\
\hline 38 & MG & $\mathbf{m}$ & 49 & 1.53 & AS; NCA (op.) & $\mathbf{0}$ & + & & 15.8 & 15.7 & 15.1 \\
\hline 39 & MC & $\mathrm{m}$ & 46 & 1.88 & $\mathrm{AI} ; \mathrm{AS}$ & $\mathbf{0}$ & + & & 18.4 & 18.3 & 17.0 \\
\hline 40 & $\mathrm{CB}$ & $\mathrm{m}$ & 44 & 1.96 & AS; NCA (op.) & 0 & + & 18.6 & 19.1 & 19.4 & 18.7 \\
\hline 41 & MN & $\mathrm{f}$ & 45 & 2.04 & PO & 0 & + & 19.8 & 20.0 & 20.5 & \\
\hline 42 & RR & $\mathrm{m}$ & 22 & 1.8 & AI ; NCA (op.) & 0 & + & & 19.8 & & 18.9 \\
\hline 43 & EY & $\mathrm{m}$ & 37 & 1.78 & AI ; AS; NCA (P.M.) & 0 & + & 19.1 & 18.5 & & \\
\hline 44 & $\overrightarrow{\mathrm{HJ}}$ & $\mathbf{m}$ & 53 & 1.74 & AI ; AS & 0 & + & 18.5 & 19.0 & 18.3 & 18.4 \\
\hline 45 & FÖ & $\mathrm{m}$ & 53 & 1.72 & MI ; NCA (op.) & 0 & + & & 18.1 & & 17.9 \\
\hline 46 & FT & $\mathrm{m}$ & 25 & 2.06 & MS (valve area $1.3 \mathrm{~cm}^{2}$ ); NCA (op.) & 0 & 0 & & 19.5 & & 19.6 \\
\hline 47 & BK & f & 23 & 1.35 & MS (valve area $1.1 \mathrm{~cm}^{2}$ ) $\mathrm{NCA}$ (op.) & 0 & 0 & & 17.2 & & 17.1 \\
\hline 48 & ELev & f & 57 & 1.47 & MS (valve area $1.3 \mathrm{~cm}^{2}$ ); NCA (op.) & 0 & 0 & 14.4 & 15.2 & 15.3 & 14.8 \\
\hline 49 & $\mathrm{HP}$ & $f$ & 56 & 1.5 & MS (valve area $0.7 \mathrm{~cm}^{2}$ ); $\mathrm{NCA}$ (op.) & 0 & 0 & 22.2 & & 22.6 & \\
\hline
\end{tabular}

* Abbreviations: $\mathrm{CI}=$ coronary insufficiency; $\mathrm{CHF}=$ congestive heart failure; $\mathrm{FSM}=$ functional systolic murmur; $\mathrm{CA}=$ coarctation of aorta $; \mathrm{MS}=$ mitral stenosis; $\mathrm{AI}=$ aortic insufficiency; $\mathrm{PDA}=$ patent ductus arteriosus ; $\mathrm{VSD}=$ ventricular septal defect $; \mathrm{p} / \mathrm{s}=$ pulmonary systemic $; \mathrm{T}$ of $\mathrm{F}=$ tetralogy of Fallot $; \mathrm{AS}=$ aortic stenosis; $\mathrm{SS}=$ subaortic stenosis; $\mathrm{ASD}=$ atrial septal defect; $\mathrm{LVH}=$ left ventricular hypertrophy; $\mathrm{SH}=$ systemic hypertension; 
II

myocardial oxygen extraction remained unchanged during exercise*

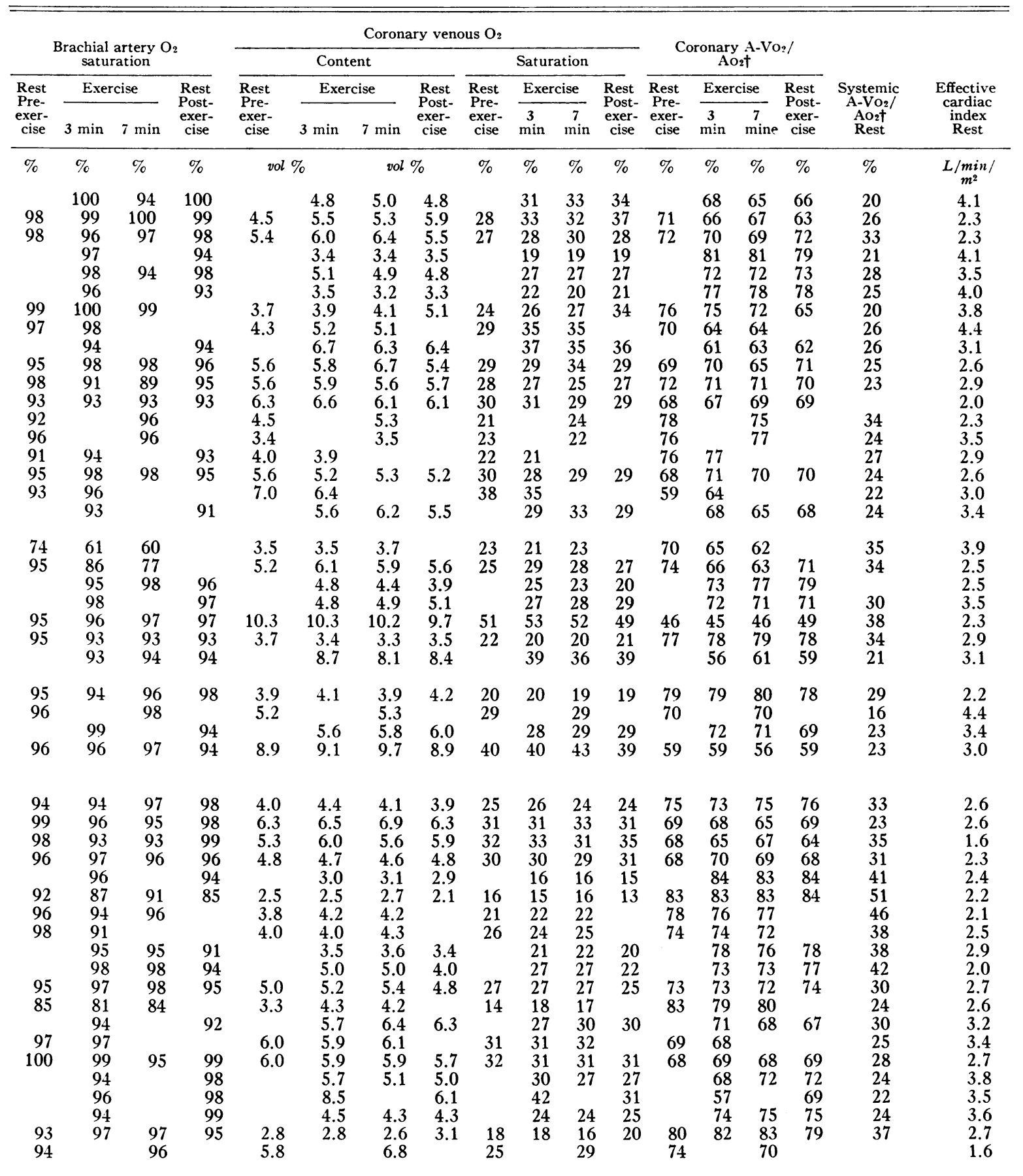

$\mathrm{SM}=$ systolic murmur $; \mathrm{CAD}=$ coronary artery disease $; \mathrm{NCA}=$ normal coronary arteries $; \mathrm{ACA}=$ abnormal ${ }^{\circ}$ coronary arteries; op. = operation; P.M. = post mortem; $\mathrm{MI}=$ mitral insufficiency; $\mathrm{PO}=$ Pickwickian obesity.

$\dagger \mathrm{A}-\mathrm{VO}_{2} / \mathrm{AO}_{2}=$ oxygen extraction percentage. 
TABLE

Clinical and physiological observations in 31 subjects in whom

\begin{tabular}{|c|c|c|c|c|c|c|c|c|c|c|c|}
\hline \multirow[b]{3}{*}{ No. } & \multirow[b]{3}{*}{$\mathrm{Pt}$} & \multirow[b]{3}{*}{ Sex } & \multirow[b]{3}{*}{ Age } & \multirow[b]{3}{*}{ BSA } & \multirow[b]{3}{*}{ Diagnosis } & \multirow[b]{3}{*}{ CI } & \multirow[b]{3}{*}{$\mathrm{CHF}$} & \multicolumn{4}{|c|}{ Brachial artery $\mathrm{O}_{2}$ content } \\
\hline & & & & & & & & \multirow{2}{*}{$\begin{array}{l}\text { Rest } \\
\text { Pre- } \\
\text { exer- } \\
\text { cise }\end{array}$} & \multicolumn{2}{|c|}{ Exercise } & \multirow{2}{*}{$\begin{array}{l}\text { Rest } \\
\text { Post- } \\
\text { exer- } \\
\text { cise }\end{array}$} \\
\hline & & & & & & & & & $3 \min$ & $7 \min$ & \\
\hline & & & yrs & $m^{2}$ & & & & \multicolumn{2}{|c|}{ vol \% } & \multicolumn{2}{|c|}{ vol \% } \\
\hline 1 & $\mathrm{RF}$ & $\mathrm{m}$ & 18 & 1.96 & HHS & 0 & 0 & 16.1 & 16.0 & 15.5 & 16.0 \\
\hline 2 & GW & $\mathrm{f}$ & 31 & 1.46 & CHB? Etiology & 0 & 0 & 16.4 & 16.7 & 16.5 & 16.1 \\
\hline$\overline{3}$ & TK & $\mathrm{f}$ & 42 & 1.75 & $\mathrm{AI} ; \mathrm{MI}$ & 0 & 0 & & 18.1 & 18.1 & 17.8 \\
\hline 4 & $J P i$ & $\mathrm{~m}$ & 46 & 1.96 & CAD; $\mathrm{ACA}(\mathrm{CA})$ & + & 0 & 19.3 & 18.9 & 18.8 & 19.4 \\
\hline 5 & $\mathrm{~J} P$ & $\mathrm{~m}$ & 55 & 1.74 & CAD & + & 0 & & 18.9 & & 18.5 \\
\hline 6 & JL & $\mathrm{m}$ & 48 & 1.69 & CAD; ACA (P.M.) & + & 0 & & 19.7 & & 19.5 \\
\hline 7 & LL & $\mathrm{f}$ & 47 & 1.51 & CAD; ACA (op.) & + & 0 & & 17.5 & 17.7 & 16.9 \\
\hline 8 & $\mathrm{~EB}$ & f & 55 & 1.46 & CAD & + & 0 & & 14.9 & & 15.0 \\
\hline 9 & WP & $\mathrm{m}$ & 50 & 1.88 & CAD; ACA (op.) & + & 0 & 20.6 & 19.8 & 20.5 & 20.4 \\
\hline 10 & FMo & f & 45 & 1.43 & CAD & + & 0 & 15.7 & 15.7 & 16.1 & 15.4 \\
\hline 11 & $\mathrm{RC}$ & $\mathbf{m}$ & 56 & 1.86 & CAD & + & 0 & 18.9 & 19.3 & 18.8 & 18.9 \\
\hline 12 & YSt & f & 52 & 1.46 & CAD; ACA (op.) & + & 0 & 17.8 & 17.4 & & \\
\hline 13 & PB & $\mathrm{f}$ & 39 & 1.60 & $\mathrm{CAD} ; \mathrm{ACA}(\mathrm{CA})$ & + & 0 & & 18.0 & & 16.9 \\
\hline 14 & JD & $\mathrm{m}$ & 51 & 1.75 & CAD; acute M.Infarct. & + & 0 & 21.0 & 22.2 & & 22.0 \\
\hline 15 & WM & $\mathrm{m}$ & 46 & 2.06 & CAD; ACA (CA; P.M.) & + & 0 & 20.2 & 20.5 & 20.1 & \\
\hline 16 & MC & f & 38 & 1.54 & $\begin{array}{l}\text { CAD; acute M.Infarct; MS (area }= \\
\left.1.1 \mathrm{~cm}^{2}\right)\end{array}$ & + & 0 & 15.8 & 16.0 & 16.3 & 15.8 \\
\hline 17 & $\mathrm{LN}$ & $\mathrm{m}$ & 43 & 1.61 & CAD; ACA (CA) & + & 0 & 17.1 & 17.7 & 17.8 & 16.8 \\
\hline 18 & $\mathrm{FM}$ & $\mathrm{m}$ & 38 & 1.68 & AS (valve area $0.8 \mathrm{~cm}^{2}$ ); NCA (op.) & + & 0 & 18.5 & 18.7 & & \\
\hline 19 & $\mathrm{JK}$ & $\mathbf{m}$ & 29 & 2.02 & AS (valve area $\left.0.8 \mathrm{~cm}^{2}\right) ; \mathrm{NCA}$ (op.) & + & 0 & 20.7 & 21.3 & 21.4 & 20.1 \\
\hline 20 & MJ & $\mathrm{f}$ & 43 & 1.61 & Tri. of $F$. & + & 0 & 18.9 & 16.3 & & \\
\hline 21 & $\mathrm{RL}$ & $\mathrm{m}$ & 40 & 2.29 & AS; AI & + & + & & 21.3 & & 21.1 \\
\hline 22 & $\mathrm{HA}$ & $\mathrm{m}$ & 56 & 1.86 & CAD & + & + & 19.2 & 19.6 & 19.2 & 19.6 \\
\hline 23 & $\mathrm{RF}$ & $\mathrm{m}$ & 47 & 1.62 & CAD; ACA (P.M.) & + & + & & 16.5 & & 15.7 \\
\hline 24 & BS & f & 45 & 1.46 & CAD; MI & + & + & 18.4 & 19.0 & 19.5 & \\
\hline 25 & LM & $\mathrm{m}$ & 51 & 1.56 & $\mathrm{AI} ; \mathrm{AS}$ & + & + & 16.9 & 17.5 & 17.3 & 16.9 \\
\hline 26 & MH & f & 55 & 1.80 & MI & 0 & + & 15.2 & 15.9 & 16.3 & 15.4 \\
\hline 27 & RH & $\mathrm{m}$ & 28 & 2.0 & AI; MI ; NCA (P.M.) & 0 & + & 15.6 & 16.2 & 16.4 & 16.2 \\
\hline 28 & JFol & $\mathrm{m}$ & 37 & 1.88 & MS (valve area $0.9 \mathrm{~cm}^{2}$ ); NCA (P.M.) & + & 0 & 20.2 & 21.1 & & 20.2 \\
\hline 29 & GR & f & 30 & 1.4 & MS (valve area $0.6 \mathrm{~cm}^{2}$; NCA (P.M.) & + & 0 & & 20.6 & & 19.6 \\
\hline 30 & $\mathrm{RB}$ & $\mathrm{f}$ & 33 & 1.51 & MS (valve area $\left.0.9 \mathrm{~cm}^{2}\right) ; A S$ & + & 0 & 13.6 & 14.3 & 14.5 & 14.4 \\
\hline 31 & $\mathrm{AH}$ & $\mathrm{m}$ & 47 & 1.84 & MS (valve area $1.0 \mathrm{~cm}^{2}$ ) & 0 & 0 & 16.9 & 17.7 & 17.8 & 17.2 \\
\hline
\end{tabular}

* For abbreviations, see Table II. Also: HHS = hyperkinetic heart syndrome; $\mathrm{CHB}=$ complete heart block; Tri. of F. = trilogy of Fallot; M. Infarct. = myocardial infarction; CA = coronary arteriography.

The 80 observations made during rest and exercise (Tables II and III) have been divided according to the effect of exercise on myocardial oxygen extraction percentage and coronary venous oxygen saturation. For the purpose of this grouping, a significant change during exercise was considered to be an absolute decrease of 5 per cent or greater in coronary venous oxygen saturation and an absolute increase of 3 per cent or greater in myocardial oxygen extraction percentage. These values were chosen so that all changes would exceed the experimental error of the van Slyke method in this laboratory $( \pm 0.2$ vol per cent).

In 41 of the 49 observations in Table II, resting myocardial oxygen extraction precentage and cor- onary venous oxygen saturation remained unchanged throughout exercise. ${ }^{3}$ In eight observations extraction decreased and saturation increased. The exercise pattern of myocardial oxygen extraction was unrelated to absolute resting values of extraction or coronary venous oxygen saturation.

The 31 studies exhibiting increased myocardial oxygen extraction and decreased coronary venous oxygen saturation on exercise are shown in Table III. Average myocardial oxygen extraction in

${ }^{3}$ This conclusion held regardless of whether the rest measurement preceded or followed exercise. Extraction percentage remained unchanged in 17 of 18 subjects in whom both types of rest observations were made, in 30 of 31 observations made before exercise only, and in 33 of 35 observations made after exercise only. 
III

myocardial oxygen extraction increased during exercise*

\begin{tabular}{|c|c|c|c|c|c|c|c|c|c|c|c|c|c|c|c|c|c|}
\hline \multicolumn{4}{|c|}{$\begin{array}{c}\text { Brachial artery } \mathrm{O}_{2} \\
\text { saturation }\end{array}$} & \multicolumn{4}{|c|}{$\begin{array}{c}\text { Coronary venous } \mathrm{O}_{2} \\
\text { content }\end{array}$} & \multicolumn{4}{|c|}{$\begin{array}{c}\text { Coronary venous } \mathrm{O}_{2} \\
\text { saturation }\end{array}$} & \multicolumn{4}{|c|}{ Coronary $\mathrm{A}-\mathrm{Vo}_{2} / \mathrm{AO}_{2} \dagger$} & \multirow{3}{*}{ 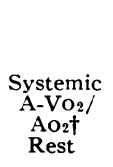 } & \multirow{3}{*}{$\begin{array}{c}\text { Effective } \\
\text { cardiac } \\
\text { index } \\
\text { Rest }\end{array}$} \\
\hline \multirow{2}{*}{ 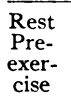 } & \multicolumn{2}{|c|}{ Exercise } & \multirow{2}{*}{$\begin{array}{l}\text { Rest } \\
\text { Post- } \\
\text { exer- } \\
\text { cise }\end{array}$} & \multirow{2}{*}{$\begin{array}{l}\text { Rest } \\
\text { Pre- } \\
\text { exer- } \\
\text { cise }\end{array}$} & \multicolumn{2}{|c|}{ Exercise } & \multirow{2}{*}{$\begin{array}{l}\text { Rest } \\
\text { Post- } \\
\text { exer- } \\
\text { cise }\end{array}$} & \multirow{2}{*}{$\begin{array}{l}\text { Rest } \\
\text { Pre- } \\
\text { exer- } \\
\text { cise }\end{array}$} & \multicolumn{2}{|c|}{ Exercise } & \multirow{2}{*}{$\begin{array}{l}\text { Rest } \\
\text { Post- } \\
\text { exer- } \\
\text { cise }\end{array}$} & \multirow{2}{*}{$\begin{array}{l}\text { Rest } \\
\text { Pre- } \\
\text { exer- } \\
\text { cise }\end{array}$} & \multicolumn{2}{|c|}{ Exercise } & \multirow{2}{*}{$\begin{array}{l}\text { Rest } \\
\text { Post- } \\
\text { exer- } \\
\text { cise }\end{array}$} & & \\
\hline & $3 \mathrm{~min}$ & $i \min$ & & & $\stackrel{3}{\min }$ & $\stackrel{7}{\min }$ & & & $\stackrel{3}{\min }$ & $\stackrel{7}{\min }$ & & & $\stackrel{3}{\min }$ & $\stackrel{7}{\min }$ & & & \\
\hline$\%$ & $\%$ & $r_{0}$ & $\%$ & vol & & & $\%$ & $\%$ & $\%$ & $\%$ & $\%$ & $\%$ & $\%$ & $\%$ & $\%$ & $\%$ & $L / \min ^{2} /$ \\
\hline 97 & 98 & 95 & 97 & 4.9 & 4.1 & 4.1 & 4.9 & 30 & 25 & 25 & 30 & 70 & 74 & 73 & 70 & 27 & 5.2 \\
\hline 100 & 98 & 97 & 96 & 4.4 & 3.8 & 2.5 & 3.5 & 27 & 23 & 15 & 21 & 73 & 77 & 85 & 78 & 32 & 3.0 \\
\hline & 99 & 99 & 100 & & 3.7 & 3.2 & 4.5 & & 20 & 17 & 26 & & 80 & 83 & 75 & 21 & 3.6 \\
\hline 99 & 96 & 95 & 100 & 8.6 & 7.9 & 7.4 & 8.5 & 44 & 40 & 37 & 43 & 56 & 58 & 61 & 56 & 23 & 2.5 \\
\hline & 77 & & 95 & & 7.1 & 6.1 & 7.2 & & 37 & 31 & 37 & & 62 & 68 & 61 & 29 & 2.3 \\
\hline & 95 & & 96 & & & 3.8 & 5.3 & & & 19 & 26 & & & 82 & 73 & & 2.5 \\
\hline & 93 & 94 & 94 & & 5.3 & 4.7 & 5.7 & & 28 & 25 & 32 & & 70 & 73 & 67 & 33 & 2.6 \\
\hline & 95 & & 97 & & 3.3 & & 3.9 & & 21 & & 25 & & 78 & & 74 & 29 & 3.4 \\
\hline 96 & 93 & 96 & 95 & 5.0 & 3.8 & 3.7 & 4.5 & 24 & 18 & 17 & 21 & 76 & 81 & 82 & 78 & 34 & 2.5 \\
\hline 98 & 96 & 98 & 96 & 5.8 & 5.3 & 4.6 & 5.4 & 36 & 32 & 28 & 34 & 63 & 66 & 71 & 65 & 35 & 2.4 \\
\hline 94 & 94 & 92 & 94 & 6.6 & 5.2 & 5.0 & 5.8 & 33 & 25 & 24 & 29 & 65 & 74 & 73 & 69 & 32 & 2.2 \\
\hline 99 & 97 & & & 6.1 & 5.3 & & & 34 & 30 & & & 66 & 70 & & & & 2.5 \\
\hline & 97 & & 94 & & 5.3 & 5.2 & 6.5 & & 28 & 28 & 36 & & 71 & 71 & 62 & 28 & 2.5 \\
\hline 90 & 94 & & 95 & 10.1 & 9.3 & 7.6 & 10.3 & 43 & 40 & 32 & 44 & 52 & 58 & 66 & 53 & 21 & 2.4 \\
\hline 96 & 96 & 94 & & 9.8 & 8.3 & 7.4 & & 47 & 39 & 35 & & 51 & 60 & 63 & & & \\
\hline 98 & 97 & 99 & 98 & 3.6 & 2.6 & 2.1 & 3.6 & 22 & 16 & 12 & 22 & 77 & 84 & 87 & 77 & 32 & 2.5 \\
\hline 97 & 98 & 99 & 95 & 8.2 & 7.2 & 5.9 & 6.9 & 46 & 40 & 33 & 39 & 52 & 59 & 67 & 59 & 23 & 3.1 \\
\hline 97 & 97 & & & 5.1 & 4.2 & & & 27 & 22 & & & 73 & 78 & & & & 3.2 \\
\hline 95 & 96 & 97 & 93 & 6.3 & 5.6 & 5.6 & 6.3 & 29 & 25 & 25 & 29 & 70 & 74 & 74 & 69 & 28 & 2.3 \\
\hline 91 & 82 & & & 3.7 & 2.2 & & & 18 & 11 & & & 81 & 86 & & & & 2.5 \\
\hline & 95 & & 98 & & 4.8 & 4.3 & 5.2 & & 21 & 19 & 24 & & 78 & 80 & 76 & 34 & 2.3 \\
\hline 97 & 93 & 93 & 98 & 5.4 & 5.0 & 4.0 & 5.1 & 28 & 24 & 19 & 26 & 73 & 75 & 79 & 74 & 30 & 2.2 \\
\hline & 95 & & 98 & & 3.2 & & 3.7 & & 19 & & 23 & & 80 & & 77 & 57 & 1.7 \\
\hline 94 & 93 & 95 & & 5.2 & 4.0 & 4.1 & & 26 & 19 & 20 & & 72 & 79 & 81 & & 41 & 1.9 \\
\hline 96 & 93 & 92 & 93 & 4.9 & 4.5 & 4.5 & 5.4 & 28 & 24 & 24 & 31 & 71 & 74 & 74 & 68 & 29 & 3.5 \\
\hline 92 & 95 & 97 & 93 & 3.7 & 3.1 & 2.6 & 3.6 & 23 & 19 & 16 & 22 & 76 & 80 & 84 & 77 & 38 & 2.0 \\
\hline 83 & 87 & 88 & 86 & 3.2 & 3.0 & 2.5 & 3.0 & 17 & 16 & 13 & 16 & 80 & 81 & 85 & 81 & 39 & 2.8 \\
\hline 94 & 98 & & 92 & 5.9 & 6.0 & 5.1 & 6.2 & 28 & 27 & 23 & 28 & 71 & 72 & 76 & 70 & 36 & 2.1 \\
\hline & 93 & & 92 & & 3.5 & 3.1 & 3.8 & & 16 & 14 & 18 & & 83 & 85 & 80 & 44 & 1.8 \\
\hline 95 & 100 & 102 & 101 & 3.6 & 3.0 & 2.4 & 3.2 & 25 & 21 & 17 & 22 & 73 & 79 & 84 & 78 & 34 & 2.8 \\
\hline 92 & 89 & 90 & 93 & 4.8 & 4.2 & 4.0 & 4.2 & 26 & 21 & 20 & 23 & 72 & 77 & 78 & 76 & 40 & 2.6 \\
\hline
\end{tabular}

$\dagger \mathrm{A}-\mathrm{Vo}_{2} / \mathrm{AO}_{2}=$ oxygen extraction percentage.

these subjects rose from 70 at rest to 74 and 76 per cent at 3 and 7 minutes of exercise, respectively. Eighteen arailable observations revealed a return to the pre-exercise resting state, despite increased myocardial oxygen extraction during exercise.

In 52 per cent of the 122 observations taken during the 80 rest-exercise studies, exercise elicited an increase in blood oxygen-carrying capacity and arterial oxygen content of $0.4 \mathrm{vol}$ per cent or greater.

\section{Clinical-physiological correlations}

Rest and exercise values for myocardial oxygen extraction and coronary venous oxygen saturation have been analyzed in the four clinical states defined earlier. The resulting correlations are presented below.

Controls: 47 subjects (Patients 1-30, Table II; $1-3$, Table III ; 1-14, Table IV). The frequency distributions of resting myocardial oxygen extraction and of coronary venous oxygen saturation are shown in Figures $3 \mathrm{~A}$ and $4 \mathrm{~A}$, with average values of $70 \pm 6$ and $29 \pm 6$ per cent, ${ }^{4}$ respectively. As shown in Figures 5A, 6A, and 7A, no correlation existed between these factors and effective cardiac index or systemic oxygen extraction (see below).

\footnotetext{
4 Values expressed as mean \pm 1 standard deviation.
} 
TABLE IV

Clinical data, cardiac indices, and coronary and systemic blood oxygen data in 29 subjects at rest

\begin{tabular}{|c|c|c|c|c|c|c|c|c|c|c|c|c|}
\hline \multirow[b]{2}{*}{ No. } & \multirow[b]{2}{*}{$\mathrm{Pt}$} & \multirow[b]{2}{*}{ Sex } & \multirow[b]{2}{*}{ Age } & \multirow[b]{2}{*}{ BSA } & \multirow[b]{2}{*}{ Diagnosis } & \multirow[b]{2}{*}{ CI } & \multirow[b]{2}{*}{ CHF } & \multicolumn{2}{|c|}{$\begin{array}{c}\text { Coronary } \\
\text { venous } \mathrm{O}_{2}\end{array}$} & \multicolumn{2}{|c|}{$\mathrm{A}-\mathrm{VO}_{2} / \mathrm{AO}_{2} \dagger$} & \multirow[b]{2}{*}{$\begin{array}{c}\text { Cardiac } \\
\text { index }\end{array}$} \\
\hline & & & & & & & & $\begin{array}{l}\text { Con- } \\
\text { tent } \\
\text { Rest }\end{array}$ & $\begin{array}{l}\text { Sat- } \\
\text { ura- } \\
\text { tion } \\
\text { Rest }\end{array}$ & $\begin{array}{l}\text { Coro- } \\
\text { nary } \\
\\
\text { Rest }\end{array}$ & $\begin{array}{l}\text { Sys- } \\
\text { temic }\end{array}$ & \\
\hline & & & yrs & $m^{2}$ & & & & vol $\%$ & $\%$ & $\%$ & $\%$ & $\underset{m^{2}}{L / \min /}$ \\
\hline 1 & TA & $\mathrm{m}$ & 34 & 1.73 & Clubbing? Etiology & 0 & 0 & 4.0 & 27 & 71 & 32 & 3.8 \\
\hline 2 & $\mathrm{RB}$ & $\mathrm{m}$ & 57 & 1.92 & Normal & 0 & 0 & 4.6 & 26 & 74 & 33 & 2.0 \\
\hline 3 & EB & $\mathrm{m}$ & 23 & 1.98 & Normal & 0 & $\mathbf{0}$ & 5.0 & 25 & 74 & 17 & 4.2 \\
\hline 4 & $\overline{\mathrm{FS}}$ & $\mathrm{m}$ & 20 & 1.95 & Normal & 0 & 0 & 5.6 & 32 & $6 \overline{6}$ & 22 & 3.0 \\
\hline 5 & $\mathrm{~PB}$ & $\mathrm{~m}$ & 20 & 1.95 & Normal & $\mathbf{0}$ & 0 & 4.8 & 25 & 75 & & 3.4 \\
\hline 6 & $\mathrm{BC}$ & $\mathrm{m}$ & 37 & 1.83 & BSM, grade ii & $\mathbf{0}$ & 0 & 4.9 & 27 & 72 & 21 & 3.4 \\
\hline 7 & CG & $\mathrm{m}$ & 22 & 1.73 & BSM, grade ii & $\mathbf{0}$ & $\mathbf{0}$ & 6.3 & 33 & 69 & 21 & 4.3 \\
\hline 8 & DB & $\mathrm{f}$ & 27 & 1.58 & FSM, grade ii & 0 & 0 & 7.1 & 38 & 63 & & 3.7 \\
\hline 9 & MD & $\mathrm{f}$ & 21 & 1.60 & ASM, grade ii & 0 & 0 & 4.5 & 23 & 76 & & 3.5 \\
\hline 10 & HS & $\mathrm{f}$ & 29 & 1.50 & Normal & 0 & 0 & 5.7 & 37 & 64 & 27 & 4.4 \\
\hline 11 & SM & $\mathrm{m}$ & 24 & 2.08 & BSM, grade ii & 0 & 0 & 5.8 & 30 & 69 & 20 & 3.9 \\
\hline 12 & $\mathrm{JT}$ & $\mathrm{m}$ & 21 & 1.75 & $\mathrm{BSM}$, grade $\mathrm{i}$ & $\mathbf{0}$ & 0 & 6.5 & 36 & 65 & 16 & 4.7 \\
\hline 13 & KK & $\mathrm{f}$ & 31 & 1.72 & Normal & 0 & 0 & 6.1 & 33 & 67 & & 3.1 \\
\hline 14 & LM & $\mathrm{f}$ & 27 & 1.60 & Normal & 0 & 0 & 3.2 & 18 & 81 & 22 & 4.1 \\
\hline 15 & IB & $\mathrm{m}$ & 36 & 1.65 & RHD; MI ; MS; AI ; LVF & + & + & 4.6 & 24 & 77 & 43 & 1.9 \\
\hline 16 & JS I & $\mathrm{m}$ & 27 & 1.81 & RHD; AI & + & + & 5.7 & 29 & 69 & & 3.4 \\
\hline 17 & AF & $\mathrm{m}$ & 24 & 1.88 & SS; NCA (P.M.) & + & + & 5.8 & 30 & 70 & & 5.0 \\
\hline 18 & WW & $\mathrm{m}$ & 54 & 1.90 & RHD; AS; AI; NCA (P.M.) & + & + & 5.7 & 30 & 68 & 20 & 4.1 \\
\hline 19 & JD & $\mathrm{m}$ & 40 & 1.92 & AS; old M. Infarct. (pre- \& post-op.) & + & + & 4.5 & 24 & 77 & & 3.1 \\
\hline 20 & LF & $\mathrm{m}$ & 48 & 1.85 & RHD: AI & + & $\begin{array}{l}0 \\
+\end{array}$ & $\begin{array}{l}4.4 \\
62\end{array}$ & $\begin{array}{l}27 \\
32\end{array}$ & $\begin{array}{l}72 \\
67\end{array}$ & 32 & $\begin{array}{l}3.0 \\
4.4\end{array}$ \\
\hline 21 & MM & $\mathrm{f}$ & 47 & 1.50 & RHD; MI ; NCA (P.M.) & + & + & $\begin{array}{l}0.2 \\
5.1\end{array}$ & $\begin{array}{l}32 \\
29\end{array}$ & $\begin{array}{l}0 / \\
71\end{array}$ & 34 & $\begin{array}{l}4.4 \\
2.4\end{array}$ \\
\hline 22 & $\mathrm{NC}$ & $\mathrm{m}$ & 20 & 1.77 & RHD; MI & + & + & 6.0 & 32 & 67 & 17 & 3.3 \\
\hline 23 & $\mathrm{CA}$ & $\mathrm{f}$ & 30 & 1.45 & RHD; MI & 0 & + & 6.0 & 33 & 66 & 28 & 3.0 \\
\hline 24 & $\mathrm{CM}$ & $\mathrm{m}$ & 30 & 1.74 & RHD; AS & + & + & 4.4 & 25 & 74 & 33 & 3.3 \\
\hline 25 & ED & $\mathrm{m}$ & 47 & 1.73 & $\mathrm{HCD}$ & 0 & + & 5.4 & 31 & 67 & 27 & 3.7 \\
\hline 26 & RL & $\mathrm{m}$ & 35 & 1.90 & RHD; AI ; MS (valve area $0.7 \mathrm{~cm}^{2}$ ) & 0 & + & 4.0 & 20 & 80 & 36 & 2.1 \\
\hline 27 & RS & $\mathrm{f}$ & 44 & 1.54 & RHD; MS (valve area $1.3 \mathrm{~cm}^{2}$ ); MI & 0 & 0 & 3.7 & 18 & 82 & & 2.8 \\
\hline 28 & WN & $\mathrm{f}$ & 46 & 1.36 & RHD; MS (valve area $0.8 \mathrm{~cm}^{2}$ ) & 0 & 0 & 3.9 & 21 & 78 & 26 & 2.8 \\
\hline & WB & $\mathrm{m}$ & 41 & 1.63 & RHD; AS; MS (mitral valve area $1.1 \mathrm{~cm}^{2}$ ) & + & 0 & 3.5 & 19 & 81 & 34 & 2.7 \\
\hline
\end{tabular}

*For abbreviations, see Tables II and III. Also: BSM = basal systolic murmur; ASM = apical systolic murmur ; $\mathrm{RHD}=$ rheumatic heart disease; $\mathrm{LVF}=$ left ventricular failure; $\mathrm{HCD}=$. hypertensive cardiovascular disease.

$\dagger \mathrm{A}-\mathrm{VO}_{2} / \mathrm{AO}_{2}=$ oxygen extraction percentage.

During exercise 91 per cent of the controls studied had no increase in myocardial oxygen extraction or decrease in coronary venous oxygen saturation. Five ( 15 per cent) of these 33 observations had decreased extraction and increased venous saturation during exercise. The three subjects (RF, GW, TK) with increased myocardial oxygen extraction during exercise had no clinical evidence of coronary insufficiency or of congestive heart failure, although patient $\mathrm{TK}$ had an equivocal Master's test without angina pectoris. In Patient GW the resting electrocardiogram showed left axis deviation, poor precordial $\mathrm{R}$ wave progression, and complete heart block. The exercise electrocardiogram was abnormal according to criteria of Master ( 7 ), but negative according to those of Mattingly and associates (8). By current clinical criteria it was impossible to diagnose or exclude coronary disease in these subjects.

Coronary insufficiency: 28 subjects (Patients 31-35, Table II ; 4-25 and 28, Table III; 15-23 and 29, Table $I V$ ). The frequency distributions of resting myocardial oxygen extraction and coronary venous oxygen saturation for 20 subjects without coexisting congestive failure or mitral stenosis are shown in Figures $3 \mathrm{~B}$ and 4B. The average values were $66 \pm 8$ and $33 \pm 7$ per cent, respectively. These values are different from the controls at the borderline of statistical significance. ${ }^{5}$ Six of 14 subjects with only coronary artery dis-

5 Using the single tail distribution of $t, 0.025<\mathrm{p}<0.05$; for the double tail distribution of $t, 0.05<\mathrm{p}<0.1$. 

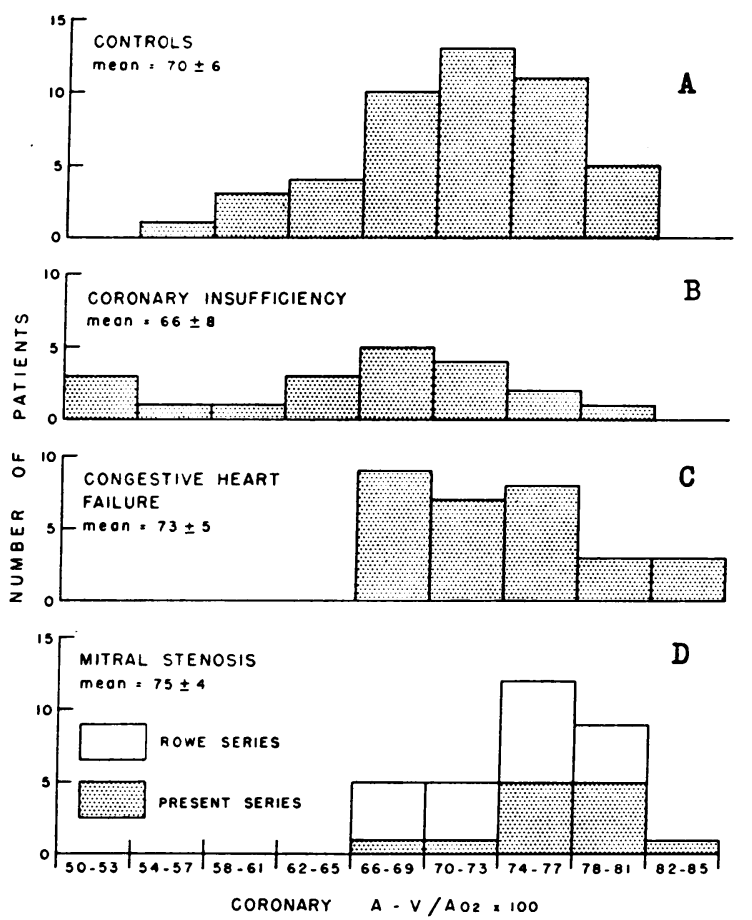

Fig. 3. FREQUENCY DISTRIBUTION OF RESTING MYOCARDIAL OXYGEN EXTRACTION PERCENTAGE FOR THE FOUR CLINICAL GROUPS. Note the lower extraction in coronary insufficiency and higher extraction in congestive failure and mitral stenosis in relation to the control group. In mitral stenosis, the open blocks are taken from Rowe and associates (16). The mean and standard deviation are shown for each group.

ease had resting oxygen extractions below 62 per cent, while this was seen in only 5 of 47 control subjects (Figure 3 ). As shown in Figures $5 \mathrm{~B}$, $6 \mathrm{~B}$ and $7 \mathrm{~B}$, no correlation existed between these factors and effective cardiac index or systemic oxygen extraction (see below).

During exercise, myocardial oxygen extraction increased and coronary venous oxygen saturation decreased in 23 ( 82 per cent) of the 28 subjects with clinical coronary insufficiency (Figure 8). The five subjects (LR, HR, LY, DH, SB) not showing this response are included in Table II. Of these , LR was a severely ill cardiac patient in whom only mild exercise could be performed because of severe fatigue. Patient HR had severe pulmonary hypertension as well as left ventricular failure.

Conversely, of the 31 subjects with increased myocardial oxygen extraction during exercise, 23
(74 per cent) had clinical coronary insufficiency. Of the remaining 8 subjects, 3 were controls (see above) and 5 had severe (class IV) valvular heart disease (Table III). The relationships between angina pectoris and myocardial oxygen patterns during exercise in all 80 observations are shown in Figure 9.

Among the 28 coronary insufficiency subjects, average myocardial oxygen extraction percentage increased from 69 at rest to 73 and 75 per cent at 3 and 7 minutes of exercise, respectively. Coronary venous oxygen saturation fell from 30 per cent at rest to 26 and 24 per cent at 3 and 7 minutes of exercise, respectively. These exercise changes are significant at the $p<0.001$ level. In 68 per cent of 22 subjects for whom data were available, progressively increasing extraction occurred during exercise. For subjects in whom data were obtained, no significant difference was observed in pre- and postexercise resting values $(0.2<p<0.4) .^{6}$ The degree of change during exercise was unrelated to absolute resting values of myocardial oxygen extraction or coronary venous oxygen saturation. Five subjects, two of whom had ischemic electrocardiographic changes, developed angina pectoris during the study. Electrocardiographic changes were seen in two other subjects without angina pectoris.

Congestive heart failure: 30 subjects $^{7}$ (Patients 34-45, Table II; 21-27, Table III; 15-26, Table $I V)$. The frequency distributions of resting myocardial oxygen extraction and coronary venous oxygen saturation are shown in Figures $3 \mathrm{C}$ and $4 \mathrm{C}$, with the average values of $73 \pm 5$ per cent and $26 \pm 5$ per cent, respectively. These values are significantly different from those of the controls $(\mathrm{p}=0.025)$.

In contrast with the control and coronary insufficiency groups, a relationship was observed between resting myocardial oxygen extraction and effective cardiac index in congestive heart failure. This relationship is shown in Figure $5 \mathrm{C}$ for the 30 resting subjects with congestive heart failure. Average myocardial oxygen extraction was 77 per

\footnotetext{
${ }^{6}$ Double tail distribution of $t$ used.

7 In order to analyze the effects of coronary insufficiency, exercise data on 7 congestive failure subjects with coexisting coronary insufficiency were included in the previous section.
} 

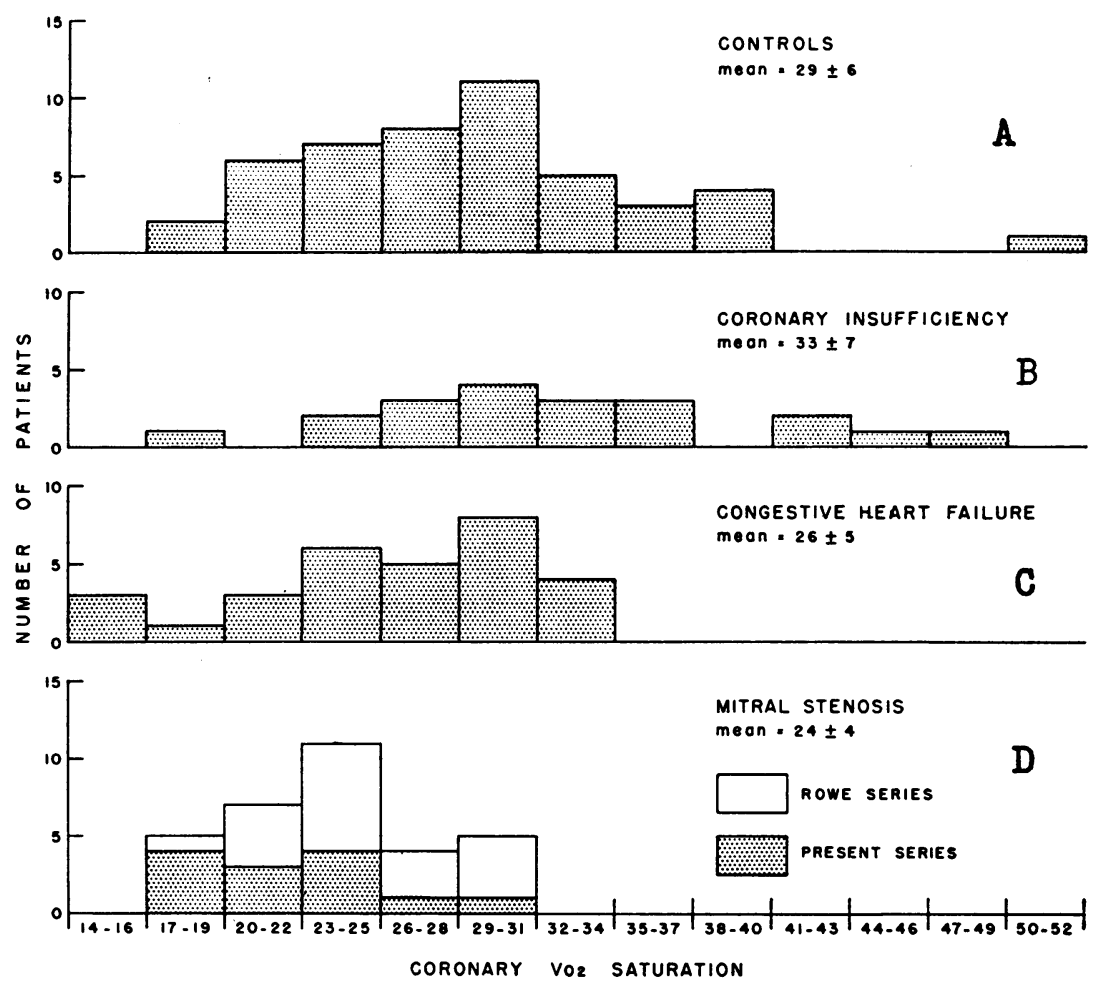

Fig. 4. Frequency distribution of Resting CORONARy venous OXYGen $\left(\mathrm{VO}_{2}\right)$ SATURATION FOR THE FOUR CLINICAL GROUPS. Note the higher saturation in coronary insufficiency and lower saturation in congestive failure and mitral stenosis in relation to the control group. In mitral stenosis, the open blocks are taken from Rowe and associates (16). The mean and standard deviation are shown for each group.

cent in subjects with cardiac indices less than 3.0, and 70 per cent for subjects with cardiac indices greater than $3.0 \mathrm{~L}$ per minute per $\mathrm{m}^{2}$. This difference is significant at the $p<0.001$ level. Subjects with cardiac indices greater than 3.0 had a frequency distribution of resting extractions similar to that in controls. Coronary venous oxygen saturation was significantly lower in subjects with cardiac indices less than 3.0 ( $p<0.001$; Figure $6 \mathrm{C})$. A positive correlation was observed between the degrees of resting oxygen extraction by the myocardium and by the body as a whole $(r=0.584 \pm 0.20$; Figure 7C). No correlation with any other hemodynamic parameter could be found (Table V).

The physiological response of the 19 exercised congestive failure subjects appeared to be related to the presence or absence of coexisting clinical coronary insufficiency. Ten of 12 failure subjects without coexisting clinical coronary insufficiency had no increase in myocardial oxygen extraction or decrease in coronary venous oxygen saturation during exercise. Thus, in the absence of coronary insufficiency, the resting "set" of myocardial oxygen extraction, whether normal or increased, was maintained during exercise. Five of seven failure subjects with coexisting coronary insufficiency had increased oxygen extraction and decreased coronary venous saturation during exercise. These seven subjects have been included in the clinical-physiological correlation for coronary insufficiency above.

Mitral stenosis: 13 subjects (Patients 46-49, Table II; 16 and 28-31, Table III; 26-29, Table $I V)$. Subjects with critical mitral stenosis (valve area $<1.5 \mathrm{~cm}^{2}$ ) have been analyzed separately because they frequently have a chronically reduced cardiac output. The frequency distributions of resting myocardial oxygen extraction and coronary venous oxygen saturation are shown in Figures 
TABLE $V$

Average hemodynamic values in the four clinical groups

\begin{tabular}{|c|c|c|c|c|c|c|c|c|c|}
\hline \multirow[b]{3}{*}{ Controls } & & $\begin{array}{l}\text { Total body } \\
\text { oxygen } \\
\text { consumption }\end{array}$ & \multicolumn{2}{|c|}{$\begin{array}{l}\text { Pressure time } \\
\text { index }\end{array}$} & \multirow{3}{*}{$\begin{array}{c}\begin{array}{c}\text { Systemic } \\
\text { arterial } \\
\text { systolic mean } \\
\text { pressure }\end{array} \\
m m \mathrm{Hg} \\
114 \pm 22 \\
133 \pm 23\end{array}$} & \multirow{3}{*}{$\begin{array}{c}\text { Heart rate } \\
\text { beats } / \text { min } \\
80 \pm 16 \\
102 \pm 17\end{array}$} & \multirow{3}{*}{$\begin{array}{c}\begin{array}{c}\text { Cardiac } \\
\text { index }\end{array} \\
L / \min / \mathrm{m}^{2} \\
3.0 \pm 0.7 \\
4.7 \pm 1.2\end{array}$} & \multirow{3}{*}{$\begin{array}{c}\begin{array}{l}\text { Coronary } \\
\text { flow }\end{array} \\
\qquad \begin{array}{c}m l / 10 \\
66 \pm 25 \\
90 \pm 45\end{array}\end{array}$} & \multirow{3}{*}{$\begin{array}{c}\begin{array}{c}\text { Myocardial } \\
\text { oxygen } \\
\text { consumption }\end{array} \\
\mathrm{g} / \min \\
7.8 \pm 2.7 \\
10.8 \pm 4.4\end{array}$} \\
\hline & & $\mathrm{ml} / \mathrm{min} / \mathrm{m}^{2}$ & \multicolumn{2}{|c|}{ mm Hg-sec } & & & & & \\
\hline & $\begin{array}{l}\mathrm{R}^{*} \\
\mathrm{E}\end{array}$ & $\begin{array}{l}129 \pm 22 \\
279 \pm 50\end{array}$ & $\begin{array}{l}2,440 \pm \\
3,360 \pm\end{array}$ & $\begin{array}{l}570 \\
780\end{array}$ & & & & & \\
\hline $\begin{array}{l}\text { Coronary } \\
\text { insufficiency }\end{array}$ & $\begin{array}{l}\mathrm{R} \\
\mathrm{E}\end{array}$ & $\begin{array}{l}137 \pm 27 \\
306 \pm 63\end{array}$ & $\begin{array}{l}2,765 \pm \\
3,800 \pm\end{array}$ & $\begin{array}{l}840 \\
860\end{array}$ & $\begin{array}{l}113 \pm 25 \\
136 \pm 33\end{array}$ & $\begin{array}{r}78 \pm 14 \\
100 \pm 16\end{array}$ & $\begin{array}{l}2.5 \pm 0.4 \\
3.5 \pm 0.9\end{array}$ & $\begin{array}{r}70 \pm 14 \\
100 \pm 27\end{array}$ & $\begin{array}{r}8.8 \pm 2.2 \\
13.8 \pm 4.1\end{array}$ \\
\hline $\begin{array}{l}\text { Congestive } \\
\text { failure }\end{array}$ & $\begin{array}{l}\mathrm{R} \\
\mathrm{E}\end{array}$ & $\begin{array}{l}153 \pm 18 \\
334 \pm 55\end{array}$ & $\begin{array}{l}3,510 \pm 1 \\
4,440 \pm 1\end{array}$ & $\begin{array}{l}1,500 \\
1,450\end{array}$ & $\begin{array}{l}115 \pm 22 \\
136 \pm 22\end{array}$ & $\begin{array}{r}85 \pm 13 \\
113 \pm 14\end{array}$ & $\begin{array}{l}2.3 \pm 0.4 \\
3.2 \pm 1.0\end{array}$ & $\begin{array}{r}91 \pm 28 \\
121 \pm 32\end{array}$ & $\begin{array}{l}11.0 \pm 4 \\
15.0 \pm 4.8\end{array}$ \\
\hline $\begin{array}{l}\text { Mitral } \\
\text { stenosis }\end{array}$ & $\begin{array}{l}\mathrm{R} \\
\mathrm{E}\end{array}$ & $\begin{array}{l}150 \pm 8 \\
341 \pm 64\end{array}$ & $\begin{array}{l}1,950 \pm \\
3,030 \pm\end{array}$ & $\begin{array}{l}550 \\
480\end{array}$ & $\begin{array}{r}98 \pm 12 \\
116 \pm 17\end{array}$ & $\begin{array}{r}88 \pm 15 \\
125 \pm 15\end{array}$ & $\begin{array}{l}2.6 \pm 0.7 \\
3.4 \pm 0.9\end{array}$ & $\begin{array}{l}68 \pm 13 \\
98 \pm 18\end{array}$ & $\begin{array}{c}9 \\
13.2 \pm 1.8 \\
2.4\end{array}$ \\
\hline
\end{tabular}

${ }^{*} \mathrm{R}=$ rest $; \mathrm{E}=$ exercise.
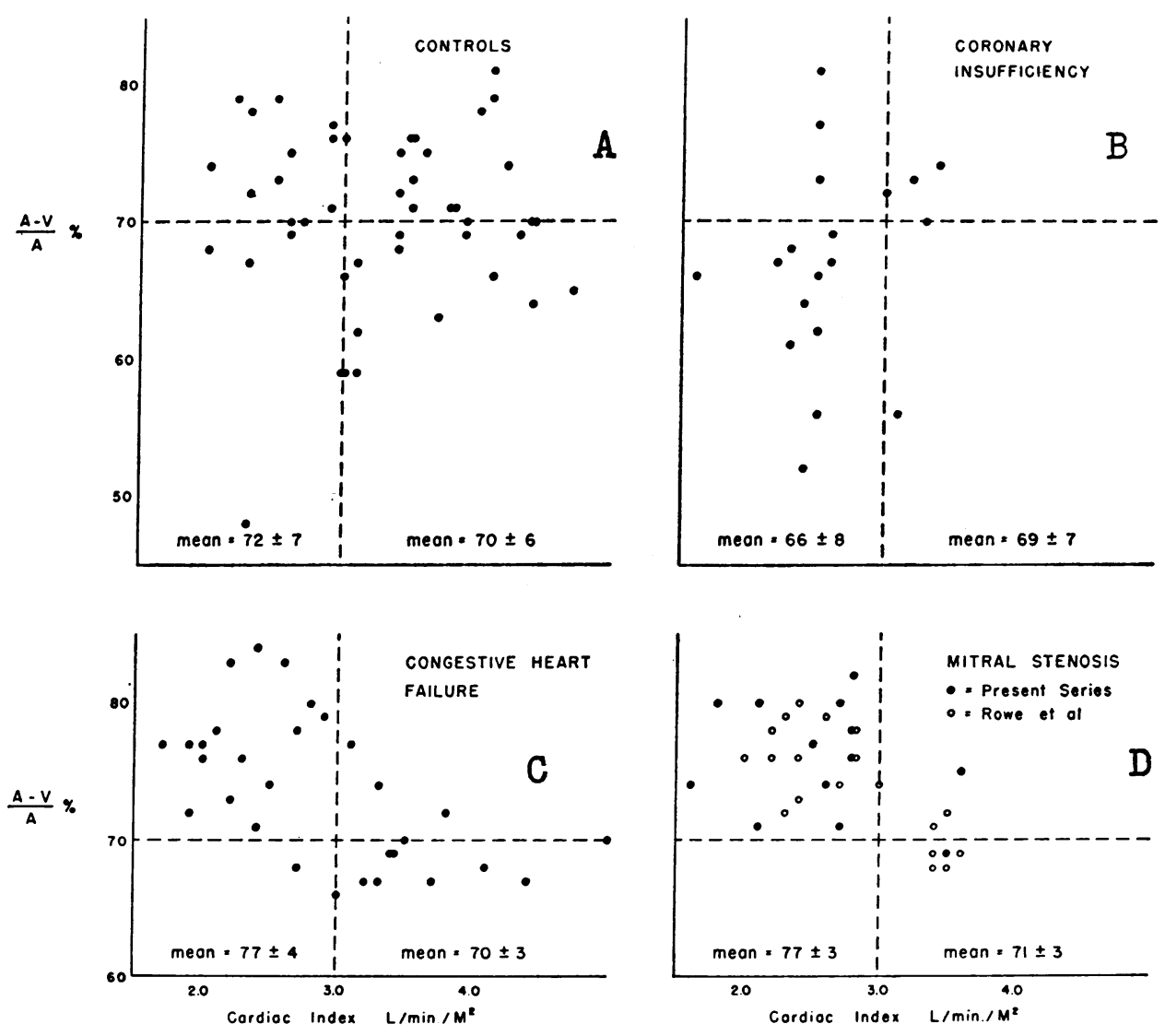

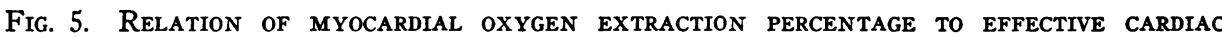
INDEX AT REST. Myocardial oxygen extraction percentage $\left(\mathrm{A}-\mathrm{Vo}_{2} / \mathrm{Ao}_{2}\right)$ is plotted against cardiac index in the four clinical groups. Note the scatter in the controls and in coronary insufficiency. In congestive heart failure and in mitral stenosis, when cardiac index was less than $3.0 \mathrm{~L}$ per minute per $\mathrm{m}^{2}$, extraction was significantly increased $(\mathrm{p}<0.001)$. The broken lines represent the average normal values for myocardial extraction and lower limits of normal for cardiac output. The mean and standard deviation of values above and below $3.0 \mathrm{~L}$ per minute per $\mathrm{m}^{2}$ are shown for each group. Open circles represent data taken from Rowe and associates (16). 

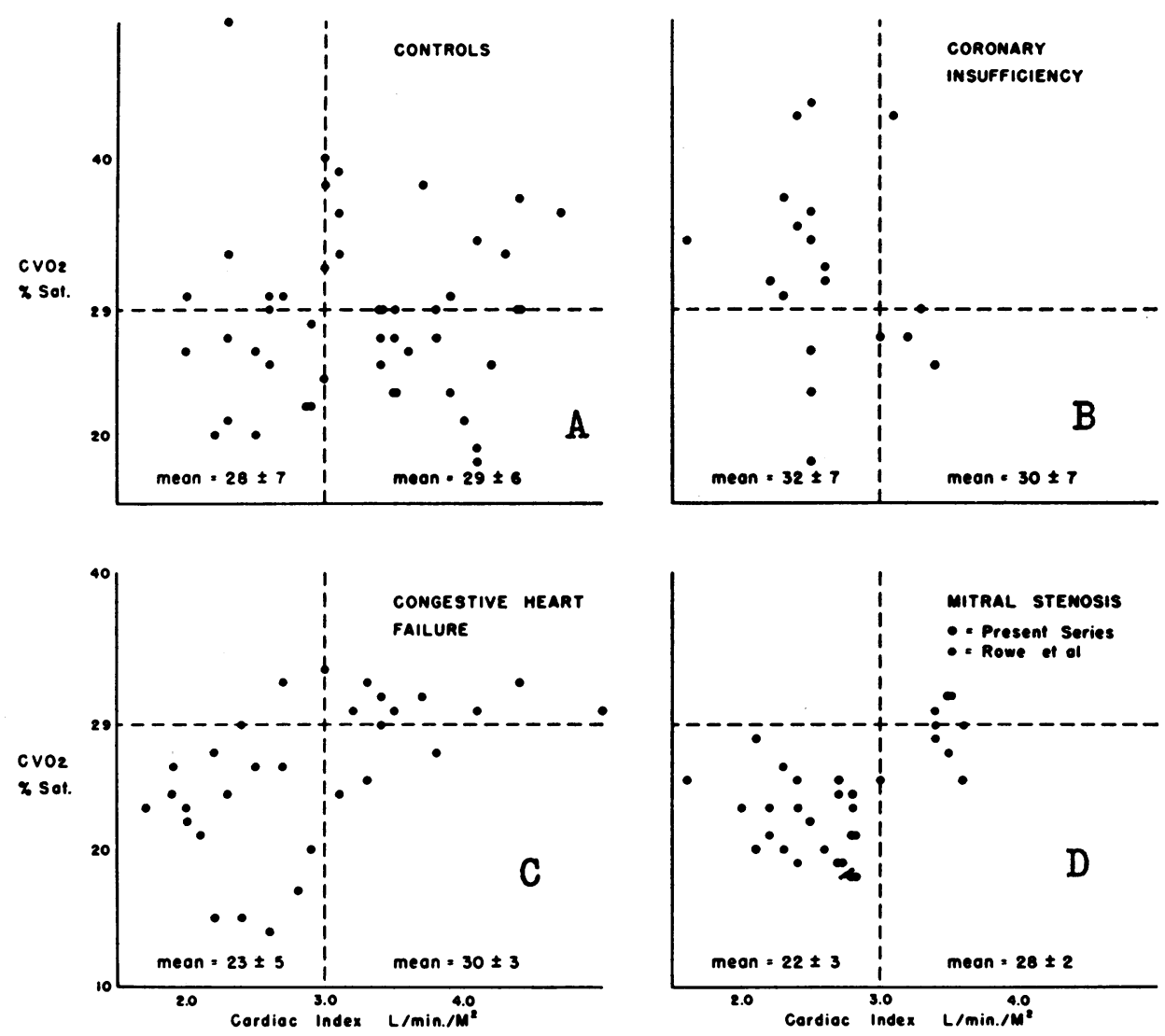

Fig. 6. RELATION OF CORONARY venOUS OXYGEN SATURATION to EFFECTIVE CARDiac indeX AT REST. Coronary venous oxygen saturation $\left(\mathrm{CVO}_{2} \%\right.$ sat.) is plotted against cardiac index in the four clinical groups. Note the scatter in the controls and in coronary insufficiency. In congestive heart failure and in mitral stenosis, when cardiac index was less than $3.0 \mathrm{~L}$ per minute per $\mathrm{m}^{2}$ saturation was significantly decreased $(\mathrm{p}<0.001)$. The broken lines represent average normal values for saturation and lower limits of normal for output. The mean and standard deviation of values above and below $3.0 \mathrm{~L}$ per minute per $\mathrm{m}^{2}$ are shown for each group. Open circles represent data taken from Rowe and associates (16).

$3 \mathrm{D}$ and $4 \mathrm{D}$, with average values of $75 \pm 4$ and

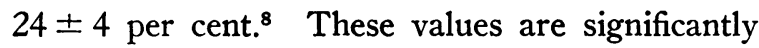
different from the controls $(p<0.001)$ but not from the congestive failure group $(0.2<\mathrm{p}<0.4)$.9

The resting relationships for mitral stenosis subjects are shown in Figures 5D, 6D, and 7D, and closely resemble those of the congestive failure group. The data in 19 mitral stenosis patients re-

${ }^{8}$ Nineteen subjects with mitral stenosis reported by Rowe and associates (16) have been included in the resting data. The authors are grateful to Dr. Rowe for providing unpublished data from these subjects.

${ }^{9}$ Double tail distribution of $t$. ported by Rowe and co-workers (16) also are included in these figures.

The physiological response of nine exercised mitral stenosis subjects was less well related to the presence or absence of coexisting coronary insufficiency than in the other clinical groups. Two subjects (MC, JFol) with coexisting coronary insufficiency have been included in the clinicalphysiological correlations for coronary insufficiency above. Of the remaining seven subjects without coexisting coronary insufficiency, four had no increase in myocardial extraction percentage or decrease in coronary venous saturation during exercise. 


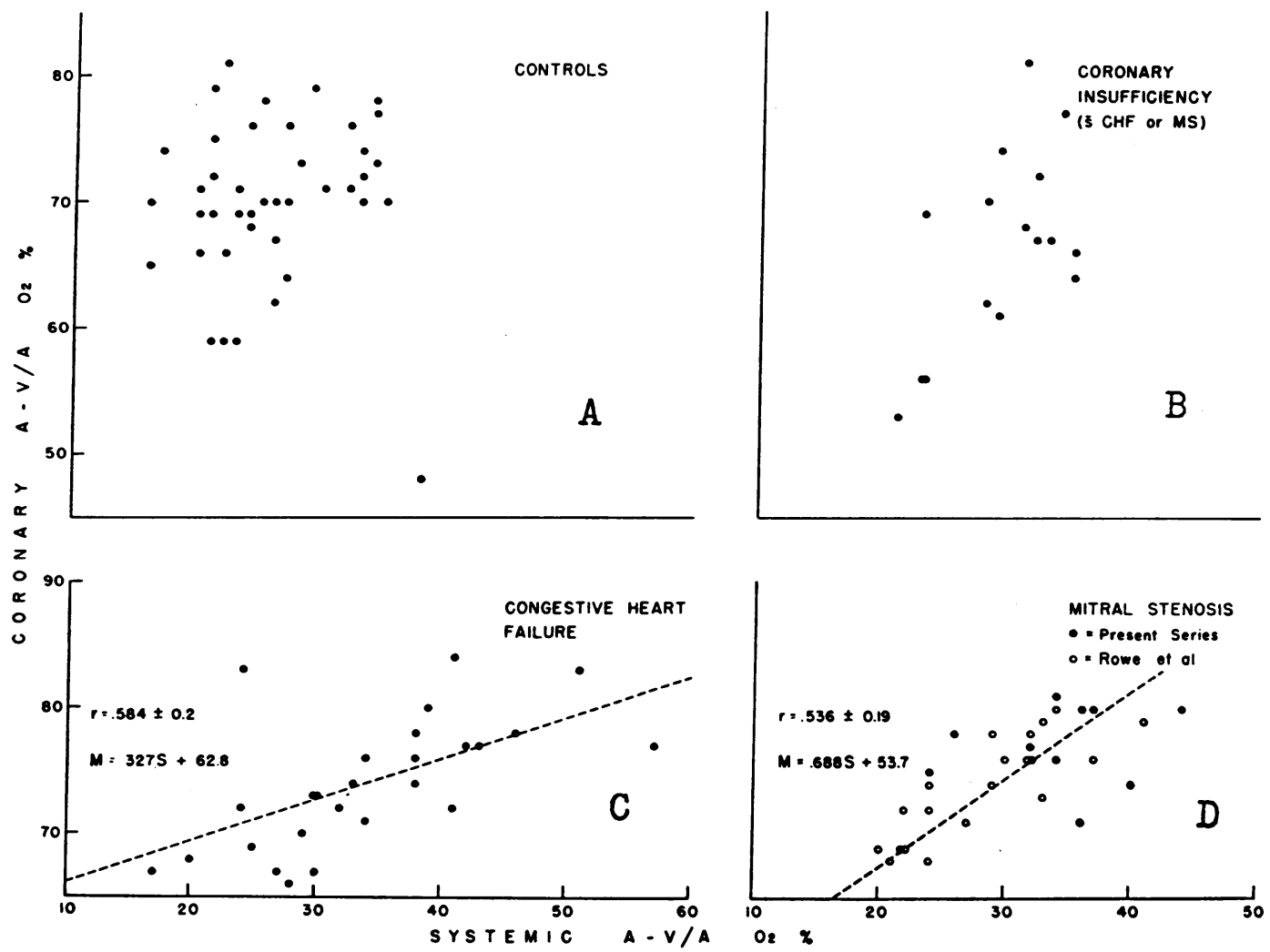

Fig. 7. Relation of myocardial to systemic oxygen extraction percentage at Rest. Myocardial oxygen extraction percentage is plotted against systemic oxygen extraction percentage. While no correlation was seen between these parameters in control or coronary insufficiency subjects, there was a correlation in subjects with congestive failure and mitral stenosis. Open circles represent data taken from Rowe and associates (16).

\section{DISCUSSION}

\section{Nature of coronary venous blood sampling}

As illustrated in Figure 1, blood entering the right atrium via the coronary sinus has several sources. The coronary sinus in man is approximately 2 inches long and receives venous blood from right and left ventricles (17-19). Beyond the entrance of the middle cardiac vein, however, nearly all blood entering the coronary sinus is of left ventricular origin. Because of possible right atrial contamination and the frequently higher oxygen saturation of middle cardiac venous blood (20), catheterization of the proximal coronary sinus or great cardiac vein was attempted in this study. Samples obtained from at least two sites suggest that homogeneous blood exists in this region of the coronary venous drainage at rest. It is of interest that this was equally so in subjects with coronary artery disease in whom heterogeneity might be anticipated (see below). In one such subject (YSt) oxygen saturations within the anterior interventricular portion of the great cardiac vein and the middle coronary sinus were essentially identical. Because of obvious technical obstacles, this homogeneity has not been confirmed during exercise.

\section{Factors affecting coronary venous oxygenation}

In the absence of significant arteriovenous shunting, coronary venous oxygenation is determined by the relationships between myocardial oxygen requirements and coronary blood flow. Factors increasing myocardial oxygen requirements-such as increased cardiac wall tension (with changes in blood pressure and cardiac volume) and heart rate, and reduced left ventricular 


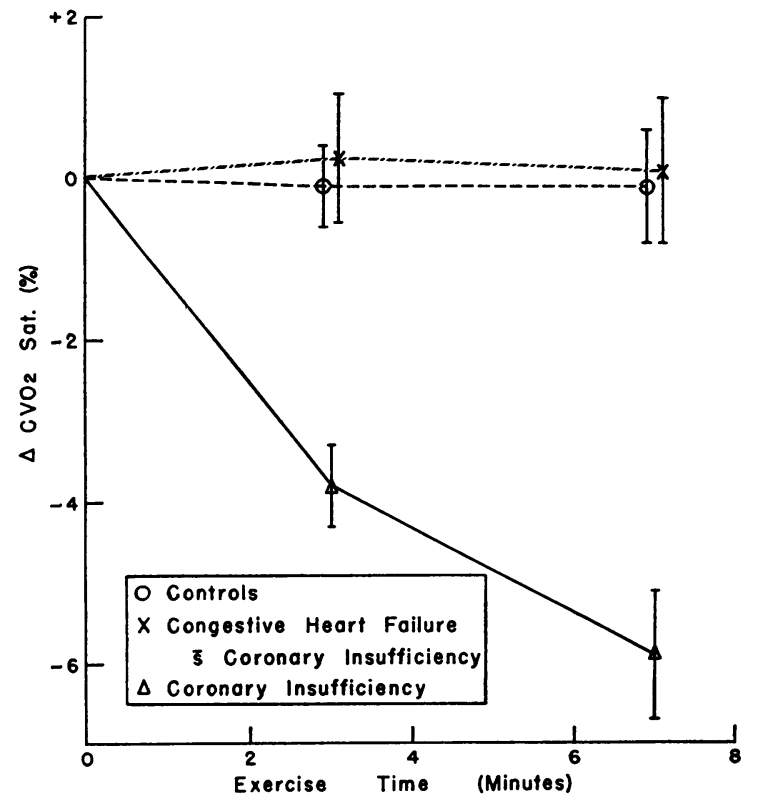

Fig. 8. REst-exercise CHANGe OF CORONARY venOUS OXYGEN SATURATION. The time course of changes in coronary venous oxygen saturation with the progression of exercise is shown for the control, coronary insufficiency, and congestive heart failure groups. The changes in coronary venous saturation of the coronary insufficiency group are significant for both the 3- and 7-minute periods $(p<0.001)$. The standard errors of the mean differences between rest and exercise observations are shown for each point in time.

efficiency-may decrease coronary venous oxygenation if not accompanied by commensurate increases in coronary blood flow. Relative inadequacy of coronary flow presumably may result from obliterative arterial disease, inadequate coronary vasodilatation, and, although not documented in man, by exhaustion of the normal reserve capacity to increase coronary blood flow during periods of extraordinary myocardial oxygen demand. Conversely, coronary blood flow in excess of myocardial oxygen requirements may produce increased coronary venous oxygenation. Such might be expected with prolonged diastole, increased aortic diastolic pressure, or inappropriate coronary vasodilatation. Arteriovenous shunting, if of significant degree, could likewise increase coronary venous oxygenation. Such shunting might, in fact, mask the underlying relationship between myocardial oxygen requirements and coronary blood flow. Homogeneity of proximal coronary sinus blood, although suggestive, does not necessarily exclude such shunting at a diffuse small vessel level.

\section{Clinical-physiological correlations}

Controls. The wide range of resting observations indicates that the relation between blood flow and oxygen extraction varies widely for the normal coronary circulation. This fact vitiates the significance of a single resting determination in assessing the adequacy of the coronary circulation. Such a value, like a single resting cardiac output, may differ greatly from the normal average.

In contrast to the heterogeneity of values observed during rest, only 9 per cent of the control subjects increased their respective resting myocardial extraction percentages during 7 minutes of exercise. Thus, in the control group, increased myocardial requirements of exercise were met almost entirely by increased coronary blood flow and slight elevation of arterial oxygen content. The arteriovenous oxygen difference rose proportionate to the rise in arterial oxygen content on exercise with preservation of the resting level of coronary venous oxygen saturation. The constancy of myocardial oxygen extraction percentage in the majority of these subjects suggests a sensitive mechanism coupling coronary blood flow to myocardial oxygen requirements, a phenomenon described in resting dogs by Eckenhoff, Hafkenschiel, Landmesser and Harmel (21). A greater

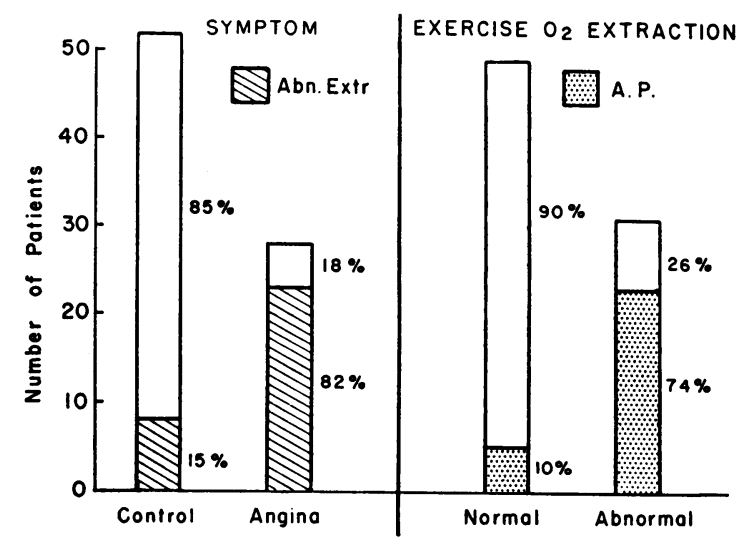

Fig. 9. Relation of ANGINa peCtoris to EXercise MYOCARDIAL OXYGEN EXTRACTION $\left(\mathrm{A}-\mathrm{Vo}_{2} / \mathrm{Ao}_{2}\right)$ PATTERNS. The relationship between clinical coronary insufficiency and abnormal myocardial oxygen extraction during exercise (see Figure 8) is shown above. Abn. Extr. =abnormal extraction; A.P. = angina pectoris. 
scatter of values than that described herein has been reported in the 12 patients with various cardiac disorders of Lombardo and associates (5) and in the 9 control subjects of Regan and colleagues (6). Their average rest-exercise myocardial oxygen extraction changes were +3 and +2 per cent, respectively.

Lactate and pyruvate values revealed no apparent anaerobic metabolism, and catecholamine levels in coronary venous blood were unchanged (20). Thus, the factor regulating blood flow was not elucidated. Possibly, changes in $\mathrm{Po}_{2}$ of the arteriolar wall or of the myocardium may influence coronary vasomotion, these studies having measured only an approximate resultant, viz., coronary venous oxygen saturation.

These observations are unique when compared with the response of skeletal muscle during exercise. Donald has documented markedly increased oxygen extraction by skeletal muscle in normal subjects during exercise (22). The resultant reduction in venous oxygenation is variable in degree, inconstant, and accompanied by excess lactic acid production. These differences undoubtedly reflect the contrasting dependencies of skeletal and heart muscle on constant levels of oxygenation.

Coronary insufficiency. Because the range of resting myocardial oxygen extraction values in this group resembled that in the controls, as reported previously $(23,24)$, resting studies have little value in assessing the adequacy of coronary vascular reserve. It is of interest that the mean value of coronary venous oxygen saturation was higher in the coronary insufficiency group than in the controls. ${ }^{10}$ This reflected the fact that 6 of 14 subjects with clinical coronary artery disease had greatly reduced oxygen extraction and increased coronary venous oxygen saturation at rest. Similar findings were alluded to by Brofman and Beck (25), and may have been seen in 2 of 8 coronary disease subjects reported by Regan and associates (26), but were not observed in four subjects studied by Rowe and colleagues (27).

The reduced resting myocardial oxygen extraction seen in some coronary disease patients has not been explained. Generalized reflex dila-

10 Using the single tail distribution of $t, 0.025<\mathrm{p}<$ 0.05 ; for the double tail distribution of $t, 0.05<\mathrm{p}<0.1$. tation of remaining normal vessels may exist in an inappropriate response to distant areas of localized myocardial ischemia. Generalized coronary vasodilatation following experimental coronary embolization has been described by Sugiura (28). In addition, arteriovenous shunts may occur through recanalized vessels located in areas of scar tissue. These mechanisms tend to increase coronary venous oxygenation. The data shown in Table I suggest that these processes, if present, are diffuse rather than localized in nature.

During exercise, myocardial oxygen extraction increased in 82 per cent of the coronary insufficiency subjects. The resultant decreases in coronary venous oxygen saturation are shown in Figure 8. Several explanations may exist for this phenomenon. Diffusely diseased coronary vessels may supply blood flow inadequate to increased myocardial oxygen demands. Increasing myocardial oxygen extraction during exercise may not be abnormal per se, but rather a normal compensatory mechanism elicited by lesser degrees of stress in coronary insufficiency than in normal subjects. As suggested earlier, part of the vascular reserve in coronary artery disease may be utilized inappropriately in the resting state by reflex vasodilatation. In congestive failure part of the reserve may be utilized appropriately to meet the commonly elevated oxygen demands of the resting state (29). When extraordinary cardiac oxygen requirements of exercise are superimposed, exhaustion of an anatomically normal coronary vasculature may result in inadequate coronary blood flow. Because of the great variability in left ventricular performance during exercise (tension development and mechanical efficiency), the magnitude of coronary blood flow change in a given subject is not necessarily indicative of flow adequate to myocardial oxygen needs. Relative coronary insufficiency, causing a decrease in coronary venous oxygen saturation on effort, can exist even with greatly increased coronary blood flow.

The observed changes may result from factors other than a blood supply inadequate to myocardial needs. If resting coronary venous oxygenation is influenced by arteriovenous shunting through recanalized vessels devoid of dilating capacity, the dominance of a fixed shunt may be overcome during exercise by increased blood flow through the remaining myocardium. Such an alteration in 
perfusion ratios might lower coronary venous oxygen saturation and cause an apparent increase in myocardial oxygen extraction. Finally, delayed coronary vasodilatation in relation to increased myocardial oxygen demands during exercise might cause temporarily increased myocardial oxygen extraction. Delayed vasodilatation could be caused by physical, metabolic or neural defects in the coronary arterioles and, if present, should become apparent in exercise studies of longer duration.

Five subjects with clinical coronary insufficiency did not increase myocardial oxygen extraction with exercise. There are several possible reasons for this. The degree of stress in this study may have been insufficient to elicit coronary vascular inadequacy in some subjects. Subject LR (Table II) is probably an example of inadequate stress. Since a coronary sinus sample represents an average of multiple areas of myocardial venous drainage, sufficiently localized disease may not affect significantly a representative sample of venous blood. Postmortem studies $(30,31)$ revealed localized coronary disease in approximately 20 per cent of patients with angina pectoris. This incidence of localized versus diffuse coronary disease is essentially identical with that of constant versus increasing myocardial oxygen extraction during exercise in subjects with coronary insufficiency (Figure 9).

The myocardial oxygen extraction changes described should be observed only when they occur within myocardium represented by the coronary venous sampling methods in this study. Right ventricular coronary insufficiency has been described by Wagman and colleagues (13). The venous drainage of this ventricle occurs via the small coronary, middle cardiac, and anterior veins, which are not represented by our sampling methods (Figure 1). These considerations may explain the apparently discrepant observation in Subject HR (Table II) who had severe pulmonary hypertension in addition to left ventricular failure.

The progressively increasing oxygen extraction observed in most coronary insufficiency subjects during exercise (Figure 8) indicates that the oxygen supply mechanism had not attained a steady state during the study period. This might result from progressively increasing myocardial oxygen consumption influenced by factors other than blood pressure and heart rate, which remained constant after 3 minutes of exercise. Alternately, this nonsteady state may have resulted from delayed attainment of capillary-myocardial equilibrium due to impaired oxygen diffusion.

Despite decreased coronary venous oxygen saturation, lactate and pyruvate values during exercise rarely revealed anaerobic metabolism in the subjects with coronary insufficiency (20). Whether reduced myocardial oxygen tension, presumably the cause of the observed reduction in coronary venous oxygen saturation, adversely affects ventricular function is not known at present. No correlation existed between electrocardiographic changes, angina pectoris, or coronary venous oxygen saturation during exercise.

The changes in coronary blood flow during exercise in subjects with coronary insufficiency were not significantly different from those in the controls (Table V). For this reason, and because of the recognized inaccuracy of the nitrous oxide coronary blood flow method, measurements of rest-exercise myocardial oxygen extraction and coronary venous oxygen saturation are of greater value in detecting physiological differences between the groups.

Congestive heart failure and mitral stenosis. In contrast with the other groups, the resting myocardial oxygen extraction in congestive failure and in critical mitral stenosis was increased in the presence of a low cardiac output. The subjects with congestive failure studied by Blain, Schafer, Siegel and Bing revealed similar findings (32). That a relative reduction in coronary flow may be indicated by increased myocardial oxygen extraction is not immediately apparent, because myocardial oxygen demands and, therefore. coronary flow were higher in congestive failure than in the controls. If, however, oxygen extraction were normal, an even higher coronary flow would be required to meet the increased oxygen needs. That increased oxygen extraction was not due to exhaustion of coronary flow reserve at rest is indicated by data reported in Table $\mathrm{V}$ which show significant elevation of coronary blood flow in these subjects during exercise.

With chronically reduced cardiac output, vasoconstriction is known to occur in many organs. Increased systemic oxygen extraction and reduced systemic venous oxygen saturation result. Increased myocardial oxygen extraction and reduced 
coronary venous oxygen saturation in congestive failure and in severe mitral stenosis indicate that the heart is not exempt from these adjustments, and suggest that the coronary circulation participates in the limitation of organ flow seen in the chronic low cardiac output state. The occurrence of this relationship in congestive failure and in mitral stenosis, and its absence in the control group, suggest that chronicity of reduced output is important in its etiology. Such patients may have chronically reduced myocardial oxygen tension at rest and in this regard resemble exercising coronary insufficiency subjects.

It is of interest that recent observations by Eisenberg, Madison and Sensenbach (33) and by Novack, Goluboff and Shenkin (34) reveal similar alterations in cerebral oxygen extraction, venous oxygenation, blood flow, and vascular resistance in low output congestive failure. Such observations require a modification of the classical concept of peripheral vasoconstriction as a protective mechanism not involving the heart and brain in chronic low output states. During exercise the pattern of myocardial oxygen extraction in congestive heart failure and in mitral stenosis appears related to the presence or absence of coexisting coronary insufficiency. Subjects with low resting cardiac outputs and no coronary insufficiency maintained a fixed elevation of myocardial oxygen extraction and fixed reduction of coronary venous oxygen saturation during exercise. This suggests that the factor(s) limiting coronary blood flow at rest in these individuals continues during exercise, competing with the vasodilator stimulus of increased myocardial oxygen demands.

\section{SUMMARY}

1. Myocardial oxygen extraction has been studied by the technic of coronary venous catheterization and measurement of multiple coronary arteriovenous oxygen differences. One hundred ten observations were made at rest and 80 during exercise.

2. During rest the control subjects had a normal frequency distribution of myocardial oxygen extraction percentages and coronary venous oxygen saturations, averaging 70 and 29 per cent, respectively. Resting values remained essentially constant throughout exercise and rest postexercise periods.

3. Subjects with coronary insufficiency had a lower mean myocardial oxygen extraction and a higher mean coronary venous oxygen saturation than did the controls at rest. During exercise myocardial oxygen extraction percentage increased and coronary venous oxygen saturation decreased significantly in 82 per cent of the subjects.

4. In congestive heart failure and in critical mitral stenosis, resting myocardial oxygen extraction percentage was increased when effective cardiac output was less than $3.0 \mathrm{~L}$ per minute per $\mathrm{m}^{2}$. During exercise the pattern of myocardial oxygen extraction appeared to be governed by the presence or absence of coexisting coronary insufficiency.

\section{ACKNOWLEDGMENT}

The statistical advice of Dr. Anthony Bartholomay is gratefully acknowledged, and the authors are indebted to Mrs. Eunice Ward and Miss Roslyn Rosenberg for their invaluable assistance.

\section{REFERENCES}

1. Goodale, W. T., and Hackel, D. B. Myocardial carbohydrate metabolism in normal dogs, with effects of hyperglycemia and starvation. Circulat. Res. 1953, 1, 509.

2. Gorlin, R., Messer, J. V., Levine, H. J., Neill, W. A., and Wagman, R. J. Coronary circulation in health and disease. Med. Clin. N. Amer. 1960, 44, 1181.

3. Case, R. B., Berglund, E., and Sarnoff, S. J. Ventricular function. VII. Changes in coronary resistance and ventricular function resulting from acutely induced anemia and the effect thereon of coronary stenosis. Amer. J. Med. 1955, 18, 39.

4. Katz, L. N., and Feinberg, H. The relation of cardiac effort to myocardial oxygen consumption and coronary flow. Circulat. Res. 1958, 6, 656.

5. Lombardo, T. A., Rose, L., Taeschler, M., Tuluy, S., and Bing, R. J. The effect of exercise on coronary blood flow, myocardial oxygen consumption and cardiac efficiency in man. Circulation 1953, 7, 71.

6. Regan, T. J., Timmis, G., Gray, M., Binak, K., and Hellems, H. K. Myocardial oxygen consumption during exercise in fasting and lipemic subjects. J. clin. Invest. 1961, 40, 624.

7. Master, A. M. Two-step exercise electrocardiogram : Test for coronary insufficiency. Ann. intern. Med. 1950, 32, 842 .

8. Mattingly, T. W., Fancher, P. S., Bauer, F. L., and Robb, G. P. The value of the double standard two- 
step exercise tolerance test detecting coronary disease in a follow-up study of 1000 military personnel. Washington, D. C., Army Medical Service Graduate School, Research Report AMSGS-2154, September, 1954.

9. Messer, J. V., Sasahara, A. A., Littmann, D. and Gorlin, R. Correlation of coronary anatomy and physiology in man (abstract). Circulation 1961, 24, 997.

10. Seldinger, S. I. Catheter replacement of the needle in percutaneous arteriography. A new technique. Acta Radiol. 1953, 39, 368.

11. Dexter, L., Whittenberger, J. L., Haynes, F. W., Goodale, W. T., Gorlin, R., and Sawyer, C. G. Effect of exercise on circulatory dynamics of normal individuals. J. appl. Physiol. 1951, 3, 439.

12. Donald, K. W., Bishop, J. M., Cumming, G., and Wade, O. L. The effect of exercise on the cardiac output and circulatory dynamics of normal subjects. Clin. Sci. 1955, 14, 37.

13. Wagman, R. J., Levine, H. J., Messer, J. V., Neill, W. A., Krasnow, N., and Gorlin, R. Coronary insufficiency in man. The physiologic and electrocardiographic correlations. Amer. J. Cardiol. 1962, 9, 439.

14. Peters, J. P., and van Slyke, D. D. Quantitative Clinical Chemistry. Vol. 2, Methods. Baltimore, Williams \& Wilkins, 1932.

15. Snedecor, G. W., and Cochran, W. G. Statistical Methods Applied to Experiments in Agriculture and Biology, 5th ed. Ames, Iowa, State College Press, 1957.

16. Rowe, G. G., Maxwell, G. M., Castillo, C. A., Huston, J. H., and Crumpton, C. W. Hemodynamics of mitral stenosis with special reference to coronary blood flow and myocardial oxygen consumption. Circulation 1960, 22, 559.

17. Gray, H. The Anatomy of the Human Body, 25th ed., C. M. Gross, Ed. Philadelphia, Lea \& Febiger, 1948.

18. Sobotta, J., and McMurrick, J. P. Atlas and Textbook of Human Anatomy. Philadelphia, W. B. Saunders, 1914, vol. 3.

19. James, T. N. Anatomy of the heart: Normal and pathologic, as related to cardiac function in Clinical Cardiopulmonary Physiology, 2nd ed., B. L. Gordon, Ed. New York, Grune \& Stratton, 1960, pp. 8-32.

20. Messer, J. V., Wagman, R. J., Levine, H. J., Neill, W. A., Krasnow, N., and Gorlin, R. Unpublished observations.

21. Eckenhoff, J. E., Hafkenschiel, J. H., Landmesser, C. M., and Harmel, M. Cardiac oxygen metabolism and control of the coronary circulation. Amer. J. Physiol. 1947, 149, 634.
22. Donald, K. W. Exercise and heart disease: A study in regional circulation. Brit. med. J. 1959, 1, 985.

23. Gorlin, R., Brachfeld, N., MacLeod, C., and Bopp, P. Effect of nitroglycerin on the coronary circulation in patients with coronary artery disease or increased left ventricular work. Circulation 1959, 19, 705.

24. Gorlin, R. Measurement of coronary flow in health and disease in Modern Trends in Cardiology, A. Morgan Jones, Ed. London, Butterworth, 1960, pp. 191-213.

25. Brofman, B. L., and Beck, C. S. Coronary heart disease. I. Hemodynamic principles and their therapeutic applications. J. thorac. Surg. 1958, 35, 232.

26. Regan, T. J., Frank, M. J., McGinty, J. F., Zobl, E., Hellems, H. K., and Bing, R. J. Myocardial response to cigarette smoking in normal subjects and patients with coronary disease. Circulation 1961, 23, 365.

27. Rowe, G. G., Chelius, C. J., Afonso, S., Gurtner, H. P., and Crumpton, C. W. Systemic and coronary hemodynamic effects of erythrol tetranitrate. J. clin. Invest. 1961, 40, 1217.

28. Sugiura, M. Experimental studies on the regulatory mechanism of the coronary circulation. Jap. Circulat. J. 1959, 23, 764.

29. Levine, H. J., Neill, W. A., Wagman, R. J., Messer, J. V., and Gorlin, R. Cardiac performance during effort in congestive failure. Clin. Res. 1960, 8, 187.

30. Blumgart, H. L., Schlesinger, M. J., and Davis, D. Studies on the relation of the clinical manifestations of angina pectoris, coronary thrombosis, and myocardial infarction to the pathologic findings, with particular reference to the significance of the collateral circulation. Amer. Heart J. 1940, 19, 1.

31. Allison, R. B., and Rodriguez, F. L. Clinicalpathological correlations in coronary artery disease. Proc. New Engl. cardiovas. Soc. 1959-60, $18,24$.

32. Blain, J. M., Schafer, H., Siegel, A. L., and Bing, R. J. Studies on myocardial metabolism. VI. Myocardial metabolism in congestive failure. Amer. J. Med. 1956, 20, 820.

33. Eisenberg, S., Madison, L., and Sensenbach, W. Cerebral hemodynamic and metabolic studies in patients with congestive heart failure. II. Observations in confused subjects. Circulation 1960 21, 704.

34. Novack, P., Goluboff, B., and Shenkin, H. A. Observations on the relationship between cardiac output and cerebral blood flow. Clin. Res. 1960, 8, 189. 